\title{
, \\ Power Quality Mitigation via Smart Demand-Side Management Based on a Genetic Algorithm
}

\author{
Adrian Eisenmann *, Tim Streubel and Krzysztof Rudion (D)
}

Citation: Eisenmann, A.; Streubel, T.; Rudion, K. Power Quality Mitigation via Smart Demand-Side Management Based on a Genetic Algorithm. Energies 2022, 15, 1492. https:// doi.org/10.3390/en15041492

Academic Editor: Miguel Castilla

Received: 27 December 2021

Accepted: 15 February 2022

Published: 17 February 2022

Publisher's Note: MDPI stays neutral with regard to jurisdictional claims in published maps and institutional affiliations.

Copyright: (C) 2022 by the authors. Licensee MDPI, Basel, Switzerland. This article is an open access article distributed under the terms and conditions of the Creative Commons Attribution (CC BY) license (https:// creativecommons.org/licenses/by/ $4.0 /)$.

\author{
Institute of Power Transmission and High Voltage Technology (IEH), 70569 Stuttgart, Germany \\ tim.streubel@ieh.uni-stuttgart.de (T.S.); rudion@ieh.uni-stuttgart.de (K.R.) \\ * Correspondence: adrian.eisenmann@ieh.uni-stuttgart.de
}

\begin{abstract}
In modern electrical grids, the number of nonlinear grid elements and actively controlled loads is rising. Maintaining the power quality will therefore become a challenging task. This paper presents a power quality mitigation method via smart demand-side management. The mitigation method is based on a genetic algorithm guided optimization for smart operational planning of the grid elements. The algorithm inherits the possibility to solve multiple, even competing, objectives. The objective function uses and translates the fitness functions of the genetic algorithm into a minimization or maximization problem, thus narrowing down the complexity of the addressed high cardinality optimization problem. The NSGA-II algorithm is used to obtain feasible solutions for the auto optimization of the demand-side management. A simplified industrial grid with five different machines is used as a case study to showcase the minimization of the harmonic distortion to normative limits for all time steps during a day at a specific grid node, while maintaining the productivity of the underlying industrial process.
\end{abstract}

Keywords: power quality; genetic algorithm; operational planning; demand-side management; multiobjective optimization; industry 4.0; fourth industrial revolution; artificial intelligence; smart grid

\section{Introduction \\ 1.1. Motivation and Idea}

Industrial processes and industrial electrical grids have been increasingly automated in recent years. Along with that, recent developments in the area of robotics have led to a high potential of demand-side integration on industrial sites. Therefore, new possibilities for a smart demand-side integration in the form of demand-side management (Smart DSM) arise and have to be investigated.

This paper is investigating the possibility and potential of an active mitigation technique to handle the PQ degradation caused by nonlinear loads in future industrial, electrical grids, without the need for conventional measures such as passive or active filtering.

The methodology follows an entirely new, user-centralized approach for an artificial intelligence (AI) guided and optimized operation of industrial plants (applying Smart DSM), based on evolutionary algorithms. Therefore, a genetic algorithm (GA) performs an optimization of the scheduling for individual nonlinear loads to actively mitigate the harmonic content in an electrical grid.

The user/plant operator first defines a schedule for the industrial process and all associated machines. If there is no mandatory condition for certain machines caused by the enclosing process, the plant operator is capable of choosing a flex option. The flex option designates the machine as a flexibility for the denominated amount of time to the GA. The GA then tries to find the global solution for the optimization problem, connected to the electrical grid. To guarantee a flawless plant operation and to shape the algorithm the least invasively, the plant operator retains the possibility to deterministically define the state of the designated machines for all time steps. The GA therefore supports the plant operator in 
finding a low-interference operating point without taking away any of the administrative options of the operator.

The optimization strategy allows different objective functions, e.g., aiming for the minimization of the total harmonic distortion (THD) of the voltage. Other options would be the mitigation of the total demand distortion (TDD) of the load current or the minimization of specific harmonics. Additionally, even incommensurable or competing objectives may be set, due to the chosen sophisticated selection scheme for the best individuals.

To sum it up, the implemented method is a proposal for deploying a possibility for an AI supported DSM method for a smart, demand-side integrated factory operation, which potentially provides a non-invasive and cost-efficient possibility of PQ mitigation without the need for an additional installation of active or passive elements such as filters.

\subsection{Literature Survey}

\subsubsection{Increasing Necessity for Power Quality Mitigation in (Industrial) Power Grids}

The waveforms of current and voltage in an electrical power grid are ideally sinusoidal and continuous, with a constant frequency and amplitude [1]. However, distorted sinusoidal waveforms of current and voltage could already be detected in the early days of electrical energy grids with alternating current around 1900. These distorted trends turned out to be a superposition of the fundamental oscillation, with multiples of this fundamental oscillation. They were subsequently called harmonics. The main sources at this time were synchronous machines, transformers and large smelting furnaces [2]. In the 1930s, interest in harmonics grew. This was partly due to the increased installation of fluorescent lamps [2] Secondly, there was inductive interference of transformer currents with the new types of wireless telephone systems [3]. Studies of transmission grids showed that the power factor of generators and loads was lower when harmonics were present. Furthermore, the third harmonic content increased from $7 \%$ at the point of connection of an electrical machine to $53 \%$ by the end of the line. This means that there was an amplification of the third harmonics within the grid. Subsequently, when asynchronous machines were examined, vibrations and scratching noises due to harmonics occurred regularly. As a result, over the next few decades, turbines were designed to be more tolerant of harmonics. In addition, a delta-star connection of the transformers prevented coupling between the transmission grid and the subordinate grids. The existing harmonics circulate in the delta windings of the transformer and are dissipated as heat loss. Due to low energy costs at the time, this was not considered a major problem [4]. The problem of the low power factor was solved from the 1950s onwards with the installation of shunt capacitors, which did not cause any problems at the time [2].

Since the beginning of the 2000s, the increased installation of renewable energy systems has also led to stability and power quality problems. These converters are equipped with control systems of different time constants, which can result in frequency coupling with the connected grid. This can lead to oscillations in a wide frequency range $[5,6]$. Such instabilities are establishing themselves as one of the main problems in the integration of renewable energy systems as well as in modern, power electronics-based industrial grid architectures $[7,8]$.

Thus far, most analyses only go up to 25th order harmonics. However, especially in the case of low-power electronic equipment, interference can also occur above these orders [3]. Moreover, most investigations concentrate on the odd harmonics solely to downsize the computational costs. Literature focussing on intermediate harmonics can be found in $[9,10]$.

The impedance of the grid is decisive for the occurrence of harmonics, resulting in resonances and instabilities. The three most important parameters in the study of harmonics in electrical grids are frequency, amplitude and phase angle. These factors are changing in modern grids mainly due to two reasons. First, the increasing installation of nonlinear and distributed power electronics in the lower voltage levels leads to more harmonic distortions and higher switching frequencies. This results in a broad frequency band in which oscillations can occur, as presented in [11]. On the other hand, the number 
of conventional resistive loads is decreasing more and more, which leads to a reduced damping of oscillations, as captured in $[12,13]$. These two effects indicate the need to analyse oscillations, especially in low-voltage grids, and to avoid them with suitable control, filtering methods or sophisticated, modern approaches such as the smart demand-side management presented in the scope of this paper.

Nowadays, the increasing number of nonlinear loads is becoming a rising concern for grid operators as well as on the consumer side, as can be understood from applied research literature [14-18]. Traditionally, passive L-C or active filters have been used for mitigating PQ problems. Disadvantages of that method are cost intensity on the one hand and the rise of probability for resonance effects in the grid on the other $[19,20]$.

State-of-the-art, custom power devices (CPD) such as active power filters (APFs) are considered to be a superior solution to the conventional passive filters to fulfill reactive power requirements and to compensate harmonics caused by nonlinear loads [21-23].

Regarding modern loads with nonlinear characteristics, light emitting diodes (LED) are gaining an increasing market share in lighting devices [24]. Therefore, the authors introduced a combination of the G1 group method and the entropy weight method to deploy a practically applicable and objective harmonic evaluation approach of harmonic emissions of LED lamps. The proposed methodology aims for a minimization of the harmonic emissions and therefore a reduced risk for fires caused by these emissions. The authors further note the transferability of the approach to other types of small commercial applications.

A bottom-up stochastic model to assess harmonic emissions in low voltage grids is presented in [25]. The model considers varying loads and their implications to the harmonic spectrum in the grid, in contrast to conventional approaches applying only stable loads. The bivariate methodology is applied to model the current harmonics via a probabilistic approach, demonstrated in a case study for the lighting load of 60 houses.

In [26], the effect of harmonic currents' cancellation on the aggregation of different load currents is evaluated to measure its impact on the grid-side by presenting a comparison between the measurement and mathematical aggregation of harmonics. Furthermore, the harmonic cancellation is also qualified for multiple loads connected to the power supply. The presented methodology was applied for an ideal sinusoidal supply voltage and odd harmonics in the range of 3 to 19.

Furthermore, a harmonic load flow framework for calculation and analysis of the propagation of harmonics in electrical grids is implemented and tested according to an easily accessible case study by the authors of this paper in [27]. This investigation deploys a simulation model for the whole PQ measurement chain, starting from a virtual PQ measurement device, including the typical configuration parameters and their correlation matrix, concluding with the propagation of the individual harmonics in the grid via a harmonic load flow model.

Following the presented information on modern day challenges in electrical grids, especially applicable for industry 4.0-bound communicative industrial grids, the detection and mitigation of harmonics is a major tool for guaranteeing grid stability and smooth operation of industrial processes. Therefore, a sophisticated AI based DSM algorithm is presented in this paper.

\subsubsection{Power Quality and Artificial Intelligence}

In the field of power quality (PQ), publications are focussing on the classification and prediction of power quality disturbances. In [28], an overview of artificial neural network (ANN) based classifiers is given. The paper points out the excessive research in the application of ANNs for power quality classification but also mentions the main drawbacks of ANNs: the strong dependence on the training data and on the hyperparameter setting. [29] investigates the effectiveness of various deep learning architectures for PQ disturbances' characterization and classification, and recommends a hybrid architecture combining convolutional neural networks (CNNs) with long short-term memory networks (LSTM). [30] 
integrates artificial intelligence techniques to forecast power quality parameters and keep them within the limits, with the aim of controlling the power flows inside an off-grid system operated under active DSM.

In [31-35], the authors of this paper conducted preliminary work on this investigation, adding classification and forecasting of PQ events to the analysis toolbox. Established machine learning approaches as well as modern methods such as LSTM were benchmarked and considered for sensitivity analyses.

Another PQ monitoring approach based on self-organizing maps (SOM) for the detection of undesirable occurrences such as sag, swell and fluctuations in the grid is presented in [36]. The authors highlight an advantage of the SOM modelling approach for preserving the topology of the data as a key feature for the detection of novel disturbances. The approach is tested by a limited, synthetic electrical signal database including four PQ disturbances-normal, sag, swell and fluctuations.

The real time ability of monitoring systems is addressed in [37], deploying an eventdriven processing, analysis and detection of $\mathrm{PQ}$ disturbances. An automatic identification of events is aimed for by the combination of two classifiers-the k-Nearest Neighbour and the Naïve Bayes. In a case study, the authors found that this approach deployed a benefit in the reduction of computational complexity as well as delays in processing and categorization of four-class PQ disturbances.

\subsubsection{Smart Grids and Reinforcement Learning}

The most commonly used reinforcement learning (RL) algorithm in smart grid applications thus far is the Q-learning algorithm. Q-learning approximates for all states of an environment how good the possible actions of an agent in each of these states are. These values are commonly trained and stored in a look-up table that contains all state-action combinations.

The appliance of Q-learning for controlling smart devices, resulting in an improvement in power quality, has been already focussed on in literature. In [38], RL algorithms were therefore applied in a four-machine power system to learn a closed-loop control law for a dynamic brake controller and a thyristor-controlled series capacitor. The objective was to avoid loss of synchronism and damp power system oscillations. The authors verified that RL methods can work without any strong assumptions on the system dynamics and learned robust closed-loop control laws. In [39], a Q-learning approach was introduced to control heterogeneous thermostatically controlled loads in order to provide short term ancillary services to the power grid. The Q-learning approach showed similar results as conventional techniques, and the authors expect that the approach can be applied on more complex extensions. In [40], the RL algorithm was suggested for usage as a secondary controller for DSTATCOM to further enhance the power quality in microgrids.

The Q-learning approach was also analysed in DSM in [41]. Motivated by the lack of an exact description of the system dynamics, a Q-learning approach was proposed for controlling a cluster of domestic electric water heaters in order to reduce the electricity cost. The RL approach is applied in [42] in a fully automated energy management system. A Q-learning based algorithm controls devices such as dishwashers, washing machines and battery controllers in order to reduce the peak load in electricity and natural gas networks and to improve the residential customers' satisfaction.

All mentioned methods fail for large-scale problems owing to the curse of dimensionality. Therefore, combining deep learning with reinforcement learning has recently had a revival of interest. Thus, in [43], the use of deep Q-learning was introduced for storage scheduling in microgrids. In [44], an online optimization of schedules for building energy management systems was implemented, analysing the use of deep Q-learning and deep policy gradient for online building energy optimization. 


\subsubsection{Genetic Algorithm Optimization}

Genetic algorithms are widely used for high complexity, global optimization problems [45-47] and even gained positive results in the field of DSM for peak demand reduction [48]. Therefore, the applicability of a GA-based PQ mitigation method shall be investigated and described in this paper.

\subsection{Contribution of the Paper}

The main objective of the paper is to develop a new mitigation approach for improving the power quality using AI-based coordination of industrial processes via Smart DSM.

The literature in Section 1.2 already confirms that AI approaches may be successfully applied for controlling devices in power systems to achieve multiple optimization goals. Despite detecting, classifying or predicting power quality disturbances, actively improving the power quality using a smart and adaptive method is becoming increasingly important. Integrating a control unit for power quality parameters in DSM by means of AI has, so far, not been investigated.

Therefore, the scope of this paper deals with the analysis of whether GA supported DSM may contribute to improving the power quality parameters in electrical, industrial grids. The additional flexibilities used for the optimization of the grid state are, in this case, the system inherent machines which are used for the industrial processing. Thus, the machine pool itself bears added value to the grid state by cancelling out one another's grid perturbations. With this new methodology, the need for costly passive or active filtering measures is reduced or, depending on the boundary conditions, even eliminated. Additionally, (physical) boundary conditions are considered via patch functions for the gene pool in the GA based optimization to increase the efficiency of the evolutionary process.

Furthermore, the proposed approach is validated using a field test-based case study in a simplified modern industrial environment (Section 3.2).

\subsection{Structure of the Paper}

The introductory part in Section 1 is followed by the problem definition and the description of the smart demand-side management methodology in Section 2. Section 2.1 characterises the industrial loads, including their load profile and their associated current characteristic. In Section 2.2, the SQL database used in this investigation is portrayed. This is followed by the introduction of the machine pool, used as flexibility in the optimization part. The innovative demand-side management algorithm used for the improvement of the grid state is described in Section 2.3. In respect to this, an HMI for the plant operator that allows the scheduling and adds a manual flexibility designation option is presented in Section 2.4. In Section 3, the results of the experiments are given. Section 3.1 shows the results for an extensive database benchmark, to evaluate the performance of different data stores for this data intensive task. Finally, Section 3.2 concludes with the validation of the methodology via a field test, implementing a simplified industrial test grid.

\section{Problem Definition and Methodology}

\subsection{Machine Pool—Virtual Twins of Industrial Loads}

There are five different types of machines: an automated guided vehicle (AGV), two industrial robots (Robot 1, Robot 2), a stacker crane and an accident proof electric saw. Table 1 presents the number of identified operation states for each machine. The discrete operation states $\Theta$ define the degrees of freedom of each machine for the optimization.

Table 1. Machines and respective number of discrete operation states $\Theta$.

\begin{tabular}{cccccc}
\hline Machine & AGV & Robot 1 & Robot 2 & El. Saw & St. Crane \\
\hline$\Theta$ & $0-4$ & $0-11$ & $0-6$ & $0-7$ & $0-5$ \\
\hline
\end{tabular}


As already stated, all machines were measured with a Class A PQ meter [49], capable of recording (transient) data with a maximal sample rate of $409.6 \mathrm{kHz}$ when triggering an event. Besides the high-resolution recording, a continuous measurement of RMS values for an averaged one second interval was conducted. All possible operating points of the industrial loads have been pre-evaluated and considered in a dedicated measurement schedule. Recorded values were the voltage $U$, the current $I$, the Power P/Q/S, the phase angles $\phi$ and the harmonic spectrum. The length of a measurement period therefore varied with the number of operating points and the time needed for a steady state to be established.

Harmonic phase angles are implemented, since they are crucial for harmonic mitigation, but the field study assumes a harmonic phase angle of $0^{\circ}$ for all machines and harmonic frequencies, resembling a worst-case scenario. Otherwise, mutual interdependencies of the machines would lower the harmonic content due to harmonic cancellation and wash out the visibility of the benefits of the optimization strategy. Nevertheless, the phase angles for all harmonic currents of the loads are included. Since each harmonic phase angle can be represented as a function of the line angle at nominal frequency, all phase angles are corrected according to Equation (1) [50]:

$$
\theta_{n}=\theta_{n-\text { spectrum }}+n \cdot\left(\theta_{1}-\theta_{1-\text { spectrum }}\right)
$$

where $n=1,2, \ldots$ represents the harmonic order, $\theta_{n}$ is the resulting phase angle of current at the grid node for the $n_{\text {th }}$ harmonic, $\theta_{n-\text { spectrum }}$ is typical phase angle of the load current for the $\mathrm{n}_{\mathrm{th}}$ harmonic, $\theta_{1}$ is the phase angle of the fundamental of current at the grid node and $\theta_{1-\text { spectrum }}$ is the typ. phase angle of the fundamental of the load current.

The two industrial robots and the electric saw work according to the state transition diagram in Figure 1c. Each of these machines has an 'off'-mode $(\Theta=0)$ and a 'standby'-mode, $\Theta=1$. The state transition diagrams show similar characteristics for Robot 1 , Robot 2 and the electric saw, with a varying number for $\Theta$.

Figures 2-4 illustrate the differences in the operation modes for the AGV, the Stacker Crane and Robot 2 via the trend line of the measured active power. Both robots can move at different speed levels. A sequence of all its movements is considered for Robot 2 with five different speed levels and for Robot 1 with ten, each as one separate operation mode. The electric saw shows five levels, characterized by an increasing rotation speed and current level. The power consumption increases with the operation modes for all machines.

Due to the change of movements within one mode for the robots, the data depict a nonsteady power consumption.

For the AGV, the charging process is considered with a total of four operation modes: the 'off' mode: $\Theta=0$, an 'idling' mode: $\Theta=1$, 'CV charging': $\Theta=2$ and 'CC charging': $\Theta=3$. Details for feasible and infeasible state transitions of the AGV are provided in Figure $1 \mathrm{~b}$. Figure 2 shows the power consumption in each operation mode. It can be seen that if the machine is connected to the charger $\Theta=3$, it consumes a large amount of power. In the constant voltage part of the charging process $\Theta=2$, the power decreases before entering an 'idling' mode $\Theta=1$, which requires only little power.

Finally, the stacker crane works according to the state transition diagram provided in Figure 1a. The characteristics of the crane depend also on the load it has to carry. Thus, $\Theta=3-5$ were measured with $20 \%, 50 \%$ and $100 \%$ load. Before operating, the machine has to wait for the user's approval, which is represented by $\Theta=2$. The power characteristics of the operation modes can be observed in Figure 3.

Additionally, to the depicted state transitions, a machine can always stay in its current mode, if it is favourable for the grid state.

Before including the flexibilities into the optimization, measurements have to be made to determine an electrical footprint of the available machine pool, thus implementing a virtual twin of the machine. Selected, characteristic features of the examined industrial loads are presented in this section. 


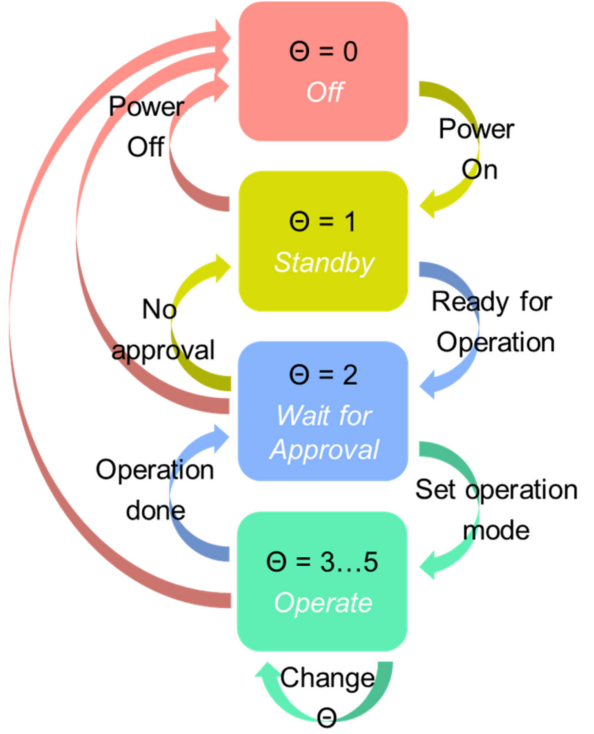

(a)

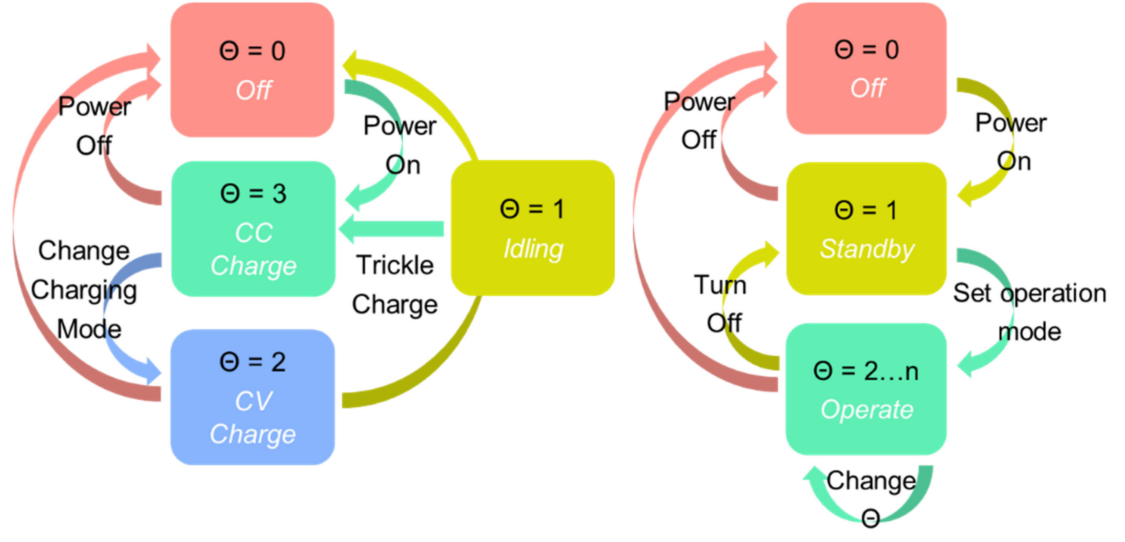

(b) (c)

Figure 1. (a) State transition diagram of the stacker crane including the decoding. To operate, the machine must pass the 'standby' mode and wait for an approval before it can switch to the 'operate' mode. If the operation is completed, the machine switches back to the 'wait for approval' mode. If no approval is given, it switches back to the 'standby' mode. (b) State transition diagram of the automated guided vehicle. It is not allowed to switch from the ' $C V$ charging' mode to the 'CC charging' mode. Another infeasible transition is to switch from 'idling' mode to the ' $C V$ charging' mode. Furthermore, to reach the ' $C V$ charging' mode starting from the 'off' mode, first the 'CC charging' mode has to be set. The 'off' mode can be accessed from all states. (c) Valid state transition diagram for the electrical saw, Robot 1 and Robot 2. Starting with the operation mode 'off', it is only allowed to switch to the operation mode 'standby'. From there, all other modes can be accessed. It is possible to jump to the 'off' mode from any other operating mode.

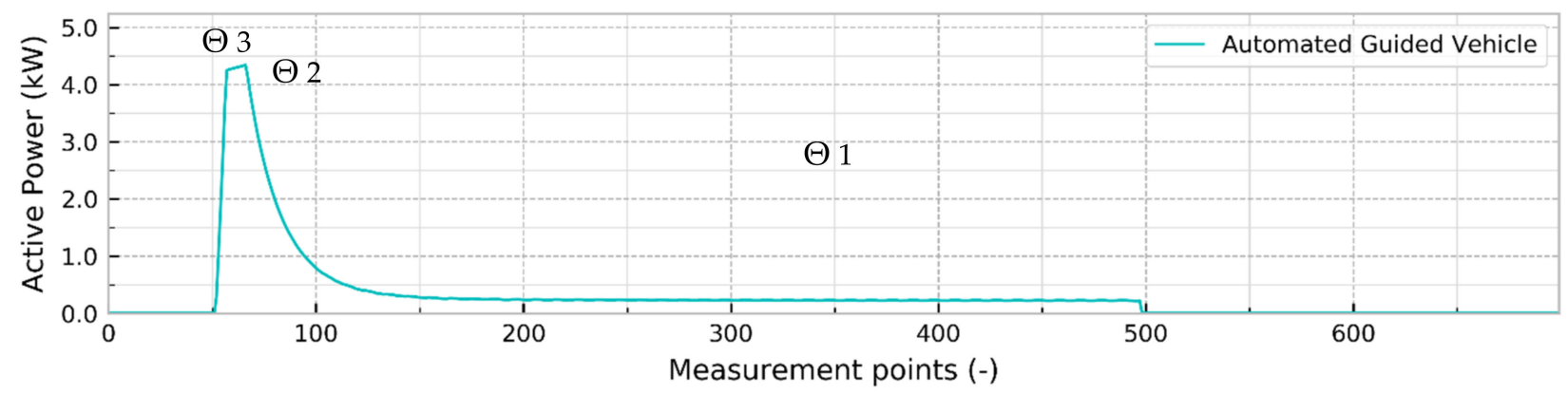

Figure 2. Time series of the power during a charging process of the AGV. $\Theta 3$ is the 'CC charging' mode, consuming the highest amount of power, $\Theta 2$ decodes the ' $C V$ charging' mode with reduced power consumption and $\Theta 1$ is the 'idling' mode. 


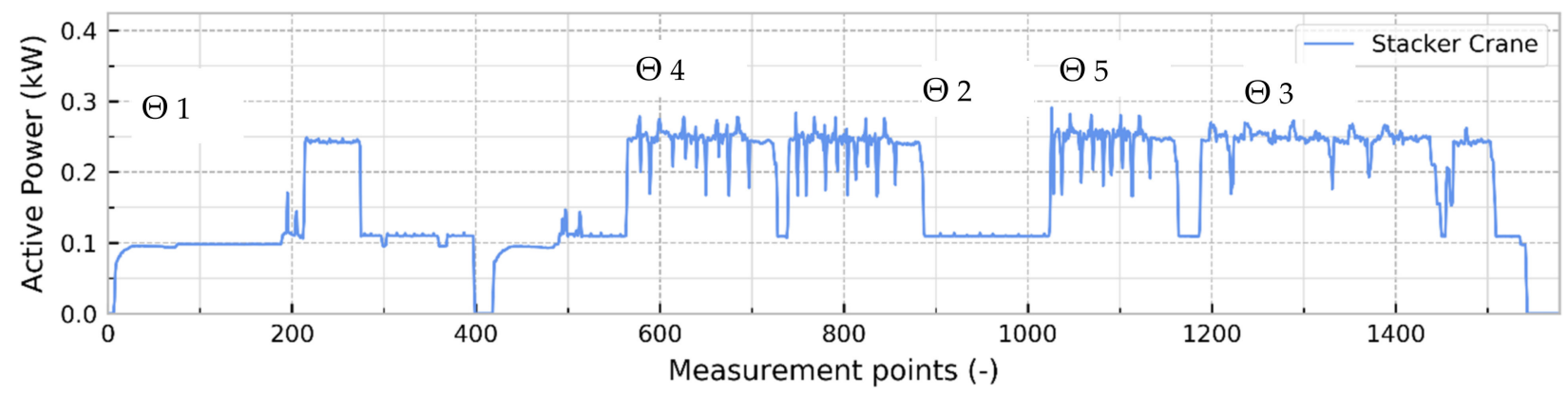

Figure 3. Time series of power for the RBG. $\Theta 1$ is the 'standby' mode, $\Theta 2$ resembles the 'wait for approval' state. In $\Theta 3-5$, the stacker crane carried $20 \%, 50 \%$ and $100 \%$ of its maximum load.

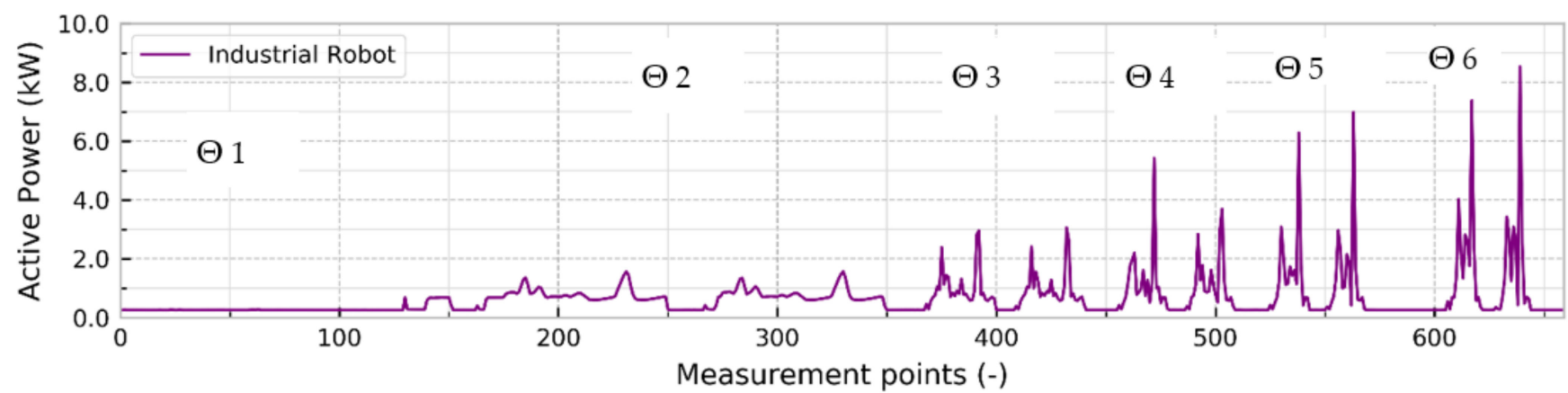

Figure 4. Time series of the sequence for the operation modes of Robot 1. During one mode, all movements of the robot are performed twice. The operation modes differ in speed. Faster movements require more power. $\Theta 1$ is the 'standby' mode, in which the machine consumes only little power. $\Theta 0$ resembles the 'off' mode, in which the machine is switched off and does not consume any power.

\subsubsection{Automated Guided Vehicle}

The driverless carrier system can be charged during its operation intervals at a charging station. For the measurement, the AGV was charged after five minutes of operation time. The charging station has a total of four different states in which the characteristics of the load differ. The measurements show standby losses of the charging station, even if the AGV is not connected. If connected, the current increases fast for the first $6 \mathrm{~s}$ and continues with a constant current $(\mathrm{CC})$ / constant voltage $(\mathrm{CV})$ characteristic until full charge. If the battery of the AGV is fully charged, the charging station switches to standby mode. Figure 5 shows the RMS values of the current trend of the charging process.

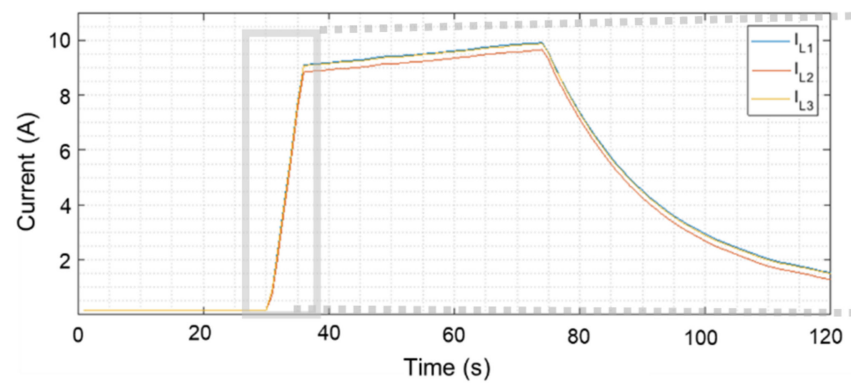

(a)

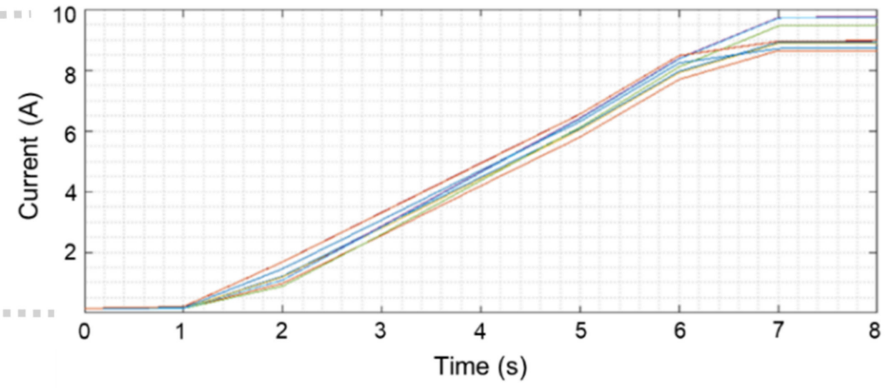

(b)

Figure 5. (a) Automated Guided Vehicle-Current trend of the AGV for a full charge cycle. (b) Rise in the RMS Value of the current during start of the charging mode. 


\subsubsection{Accident Proof Electrical Saw}

The investigated accident-proof saw is a demonstrator with a special security feature. During application, the saw blade is monitored by a camera, which immediately stops it when an undesirable obstacle is detected. The saw blade has a total of eight adjustable operating points. The measurement shows a nonlinear power consumption curve for the operating points. This characteristic is based on the transmission ratio of the gearing. In Figure 6a, a measurement cycle with the RMS values of the current signal for all three phases is depicted, including the harmonic content of the lower frequency spectrum.

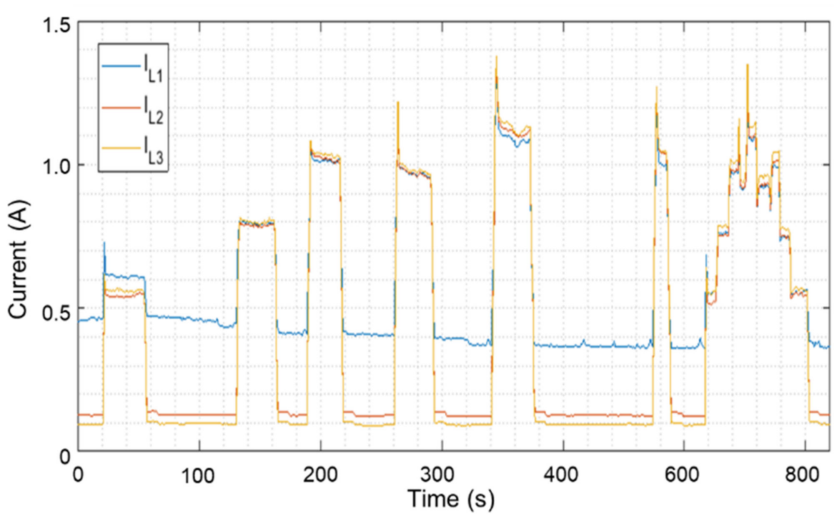

(a)

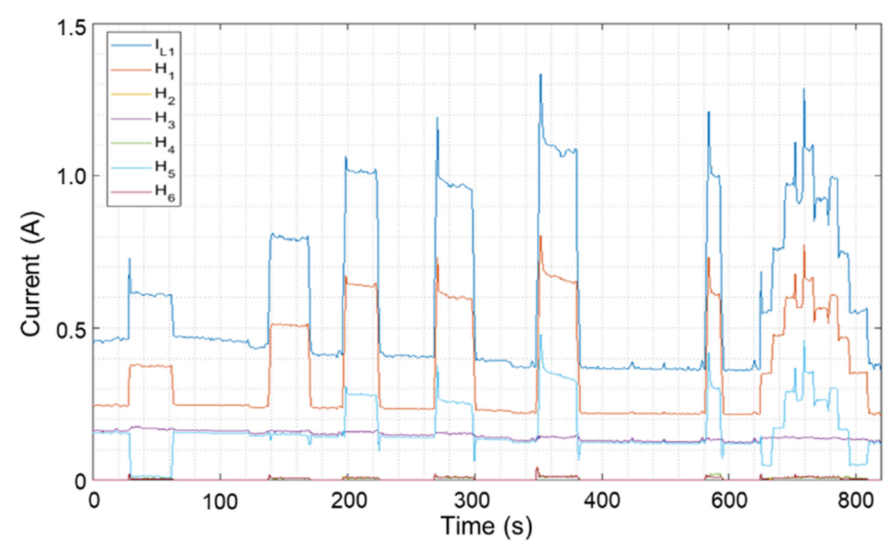

(b)

Figure 6. (a) Accident Proof Electrical Saw-Current trend of the saw for designated operation points. (b) Harmonic currents of order 1-6 for different operating points of the accident-proof saw.

In Figure $6 \mathrm{~b}$, the trends of the RMS values of the lower harmonic orders are shown to illustrate the distortion level of the measured current signal while the saw is in operation.

\subsubsection{Industrial Robot}

For the measurement of an industrial robot, a test program was written in which all axes are initially operated individually and subsequently together. This process was carried out at different speeds. The robot has a total of seven rotatable axes.

At the beginning of the test program, axis 1 rotates first in one direction, then in the opposite direction. Figure 7a shows the current trend of the first ten periods, enlarged for the first period in Figure $7 \mathrm{~b}$. It can be seen that the distortion of the current signal remains constant throughout the movement, but the maximum amplitude varies with movement speed and is up to $25 \%$ higher on average at full speed compared to a partial load of $40 \%$.

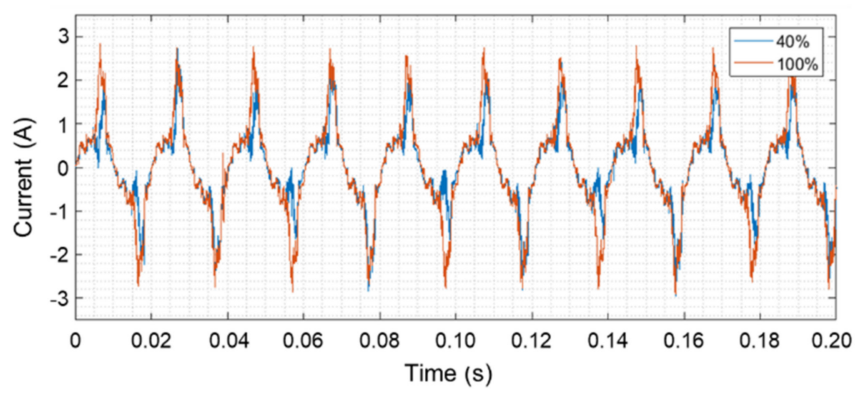

(a)

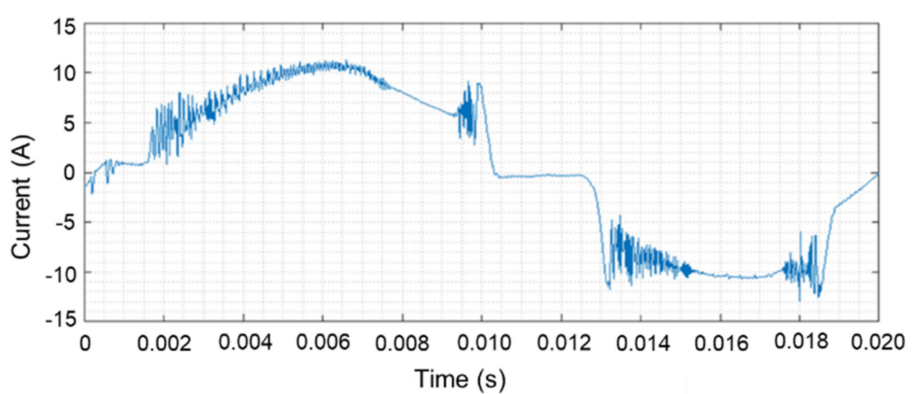

(b)

Figure 7. (a) Industrial Robot-Currents during rotation of axis 5 at $40 \%$ and $100 \%$ speed. (b) Inrush current signal of the industrial robot after activation with dominant harmonic components up to $10 \mathrm{kHz}$. 


\subsubsection{Stacker Crane}

The stacker crane has a total of three drive axes, each responsible for one direction. The storage rack can also be switched off, suspended to standby mode or run in the program sequence at normal operation. Each of the three drive axes may be controlled individually. In Figure 8a, the current at the beginning of a movement in horizontal and vertical direction is shown. The pattern of the current remains the same throughout the process, due to the switching frequency of the inverter with only the maximum amplitude changing. All three phases show the same current profile.

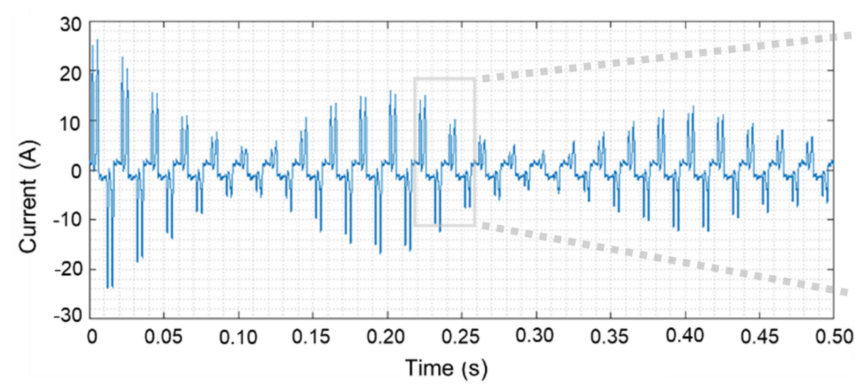

(a)

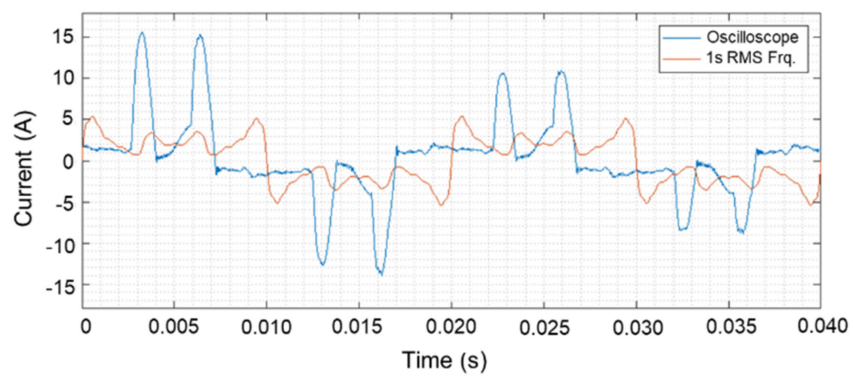

(b)

Figure 8. (a) Stacker Crane-Oscilloscope recording of the current waveform at the beginning of a translational operation of the stacker crane. (b) Current waveform from the oscilloscope record in comparison to the 1s RMS values to show the dependency of the resulting frequency spectrum in the data post processing.

In Figure $8 b$, an enlarged section of this sequence is shown. In addition, the synthesized signal from the measured 1s RMS intervals of the current harmonics is represented. Significant differences can be observed, depending on the accumulation of the data. This illustrates the need for measured high-resolution waveform data to prevent the measurement series from being misinterpreted in the post processing steps.

\subsection{Database}

In this paper, five machines and their respective operating points are analysed, namely an automated guided vehicle (AGV), an industrial robot, a stacker crane and an accidentproof electrical saw. The measured data include the harmonic content of the signal, high resolution samples $(40.96 / 409.6 \mathrm{kHz})$ and 1 s root mean square (RMS) values of voltage and current as well as the power consumption of the device.

The data were measured with a certified Class A PQ meter. Three different types of databases (DB) can be used to store the data:

1. Relational DB-Postgre $S Q L$

2. NoSQL DB, document based-MongoDB

3. Time-Series DB-InfluxDB

Figure 9 shows the entity relationship model of the PostgreSQL DB. Each measurement is connected to a machine via a one-to-one relation. Furthermore, contacts of the persons in charge are stored and connected to the machine with an $\mathrm{m}-\mathrm{n}$ relation [51]. The location of the machine is stored as well and connected to the machine with an $n-1$ relation. 


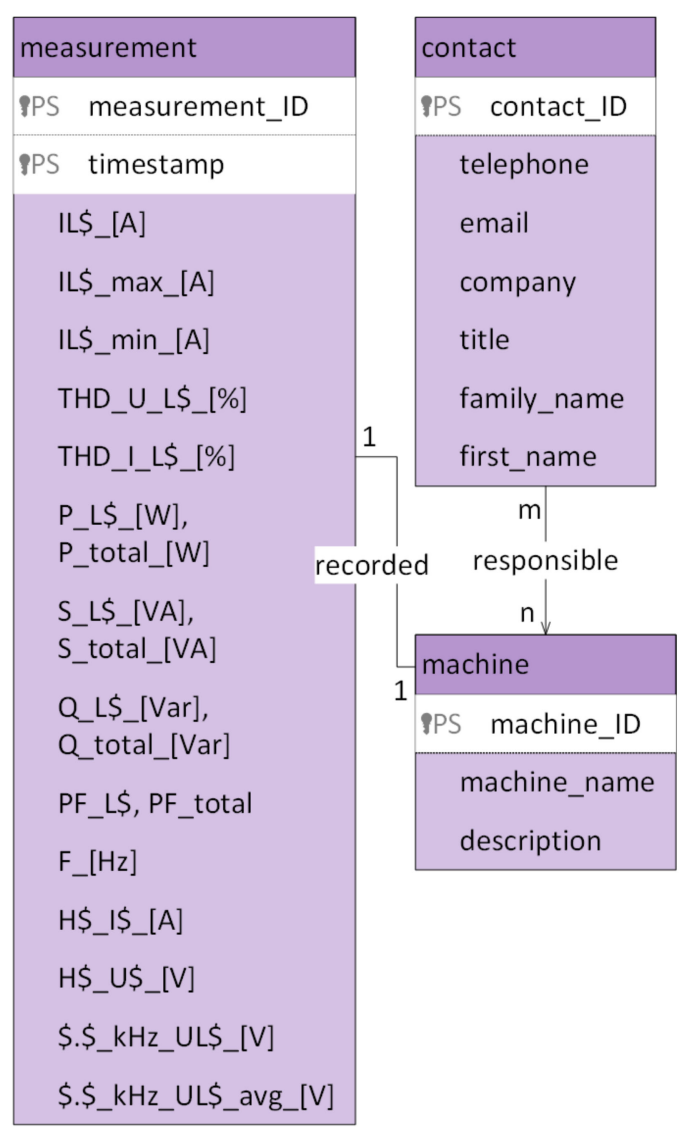

(a)

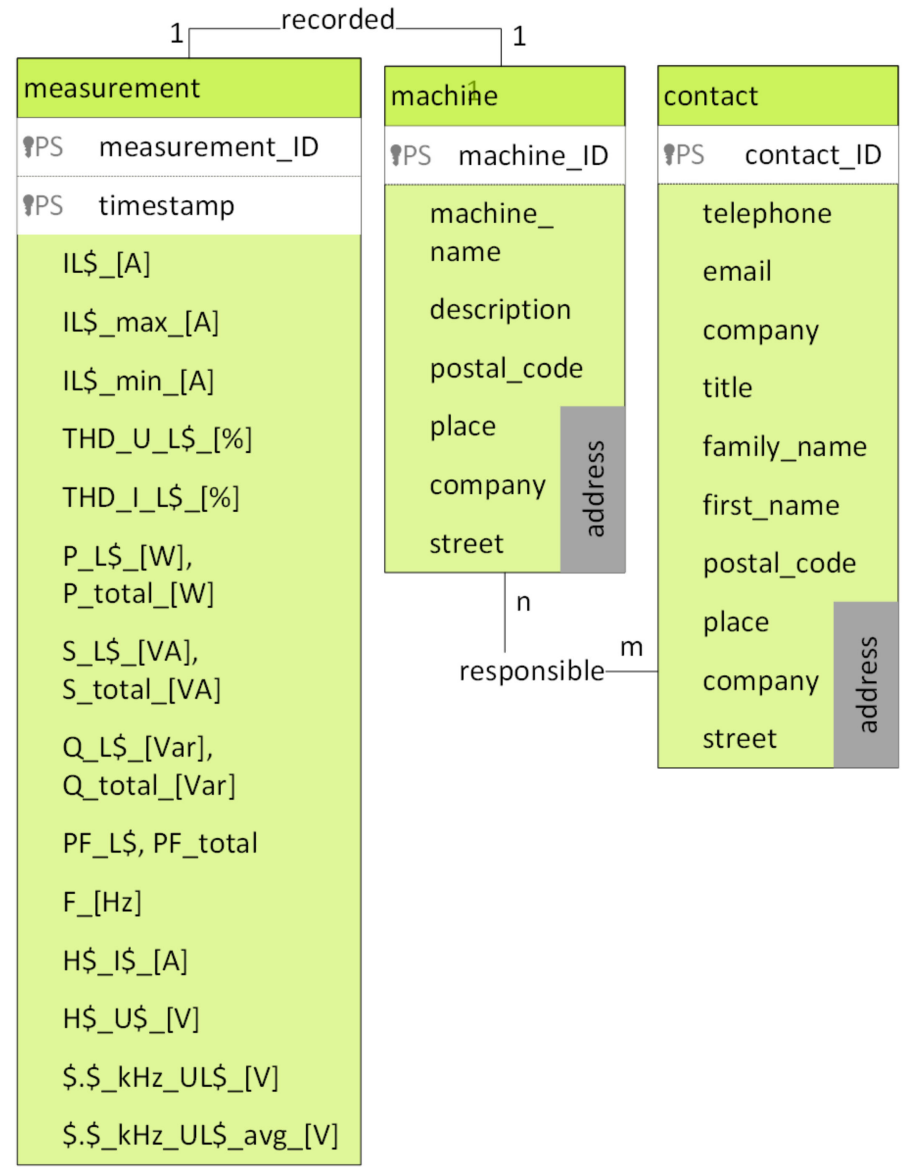

(b)

Figure 9. (a) Entity relationship model of the PostgreSQL DB. (b) Entity relationship model of the MongoDB.

Unlike relational database systems, MongoDB and document-oriented database management systems have different concepts for storing such relationships, i.e., the embedding of further documents [52,53]. For the measurement series, metadata are needed and could change. Thus, this additional machine information can be outsourced, for which reason a separate table is to be created for each machine. Doing this, relationships are not found by comparing a foreign and a primary key, rather, the documents are directly embedded into another document. Embedding automatically results in fewer read operations than outsourcing to extra documents or relationships - as in relational databases-because all information is stored together in one object [54]. For the reason of all data being queried together, the concept of embedding is used in this approach and can be seen in Figure 10.

Influx $D B$ does not support the storage of various metadata in version 1.5 and 1.6 (except for tags). A possible solution to address this problem is to outsource these data. The proposed structure is to use parts of the PostgreSQL topology and combine it with the measured time series data of $\operatorname{InfluxDB}$, as shown in Figure 10. 


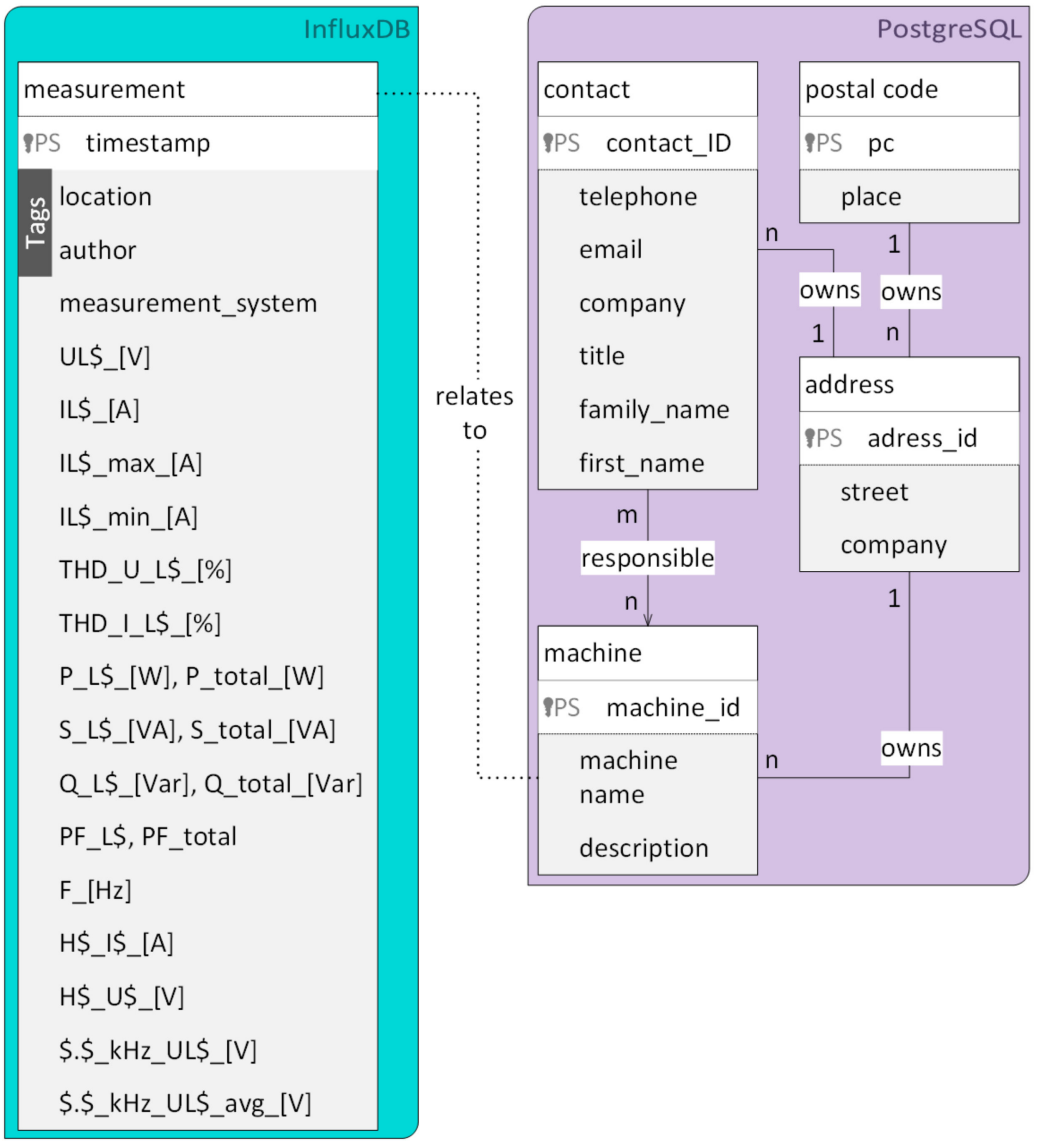

Figure 10. Combination of InfluxDB and PostgreSQL for data storage and capturing of the metadata of the stored information.

\subsection{Demand-Side Management Algorithm}

The multi-objective optimization based on the genetic algorithm is performed by invoking the evolutionary computation framework named DEAP in Python.

The Smart DSM method is a composite of three main function sets, aiming for enhanced harmonic cancellation, thus improving the PQ grid state:

(1) flexibility definition,

(2) operation optimization,

(3) feasibility check.

An HMI helps the operator to easily apply and configure the algorithm (Section 2.4). If the flexibilities of all machines that are integrated into the system are defined, the operator can perform the optimization. An overview of the simulated harmonic content and the distortion level for the resulting schedule is then provided. The optimal operating plan, harmonic contents, the power demand, the load currents and values for the THD as well as the TDD can further be obtained, visualized and saved in the database.

\subsubsection{Coding Scheme and Gene Pool for the Genetic Algorithm}

Real-value encoding is implemented in this approach. The individuals inherit the daily scheduling plans of all the machines that are integrated into the system. With $15 \mathrm{~min}$ time slots, one day can be described by 96 time units, resembled by genes with a length of 96 bytes per machine. The scheduling plan of each machine therefore can be extracted from the gene sequence. Details are shown in Table 2. The value range for the genes in each individual differs from one machine to another and varies according to the number of operation points. 
Table 2. Parameters of the Real Value Coding Scheme.

\begin{tabular}{cccc}
\hline Machine & Operating Points & Sequence in Gene & $\boldsymbol{\Theta}$ \\
\hline AGV & 4 & $0-95$ & $0-3$ \\
Robot 1 & 12 & $96-191$ & $0-11$ \\
Robot 2 & 7 & $192-287$ & $0-6$ \\
El-Saw & 8 & $288-383$ & $0-7$ \\
St. Crane & 6 & $384-479$ & $0-5$ \\
\hline
\end{tabular}

\subsubsection{Optimization Problem-Fitness Functions and Objective Function}

The $T H D_{V}$, and the $T D D_{I}$ are implemented as fitness functions for the GA to ensure a high PQ in the grid. The computation of the $T H D_{V} / T D D_{I}$ is conducted via (2). Besides the max-value, the $95 \%$ percentile, i.e., the value that is not exceeded during $95 \%$ of the time period, is used for the computation, considering the normative limits from EN 50160 [55] and IEC 61000-2-2 [56]. The limits of the $T D D_{I}$ are set in IEEE 519 [57]. The fitness function that was used to ensure a high utilization of the underlying industrial process is the drawn energy during one day $E_{96}(3)$. Nevertheless, other fitness functions can be added easily due to the straightforward implementation of the target values for the optimization in this approach. Positive tests were conducted for the minimization of specific harmonics, as well.

$$
\begin{gathered}
T H D_{V n i}=\sqrt{\frac{V_{2_{n i}}^{2}+V_{3_{n i}}^{2}+V_{4_{n i}}^{2}+\cdots+V_{50_{n i}}^{2}}{V_{1_{n i}}^{2}}} \times 100 \%=\sqrt{\frac{\sum_{k=2}^{50} V_{k_{n i}}^{2}}{V_{1_{n i}}^{2}}} \times 100 \% \\
T D D_{I n i}=\frac{\sqrt{I_{2_{n i}}^{2}+I_{3_{n i}}^{2}+\cdots+I_{50_{n i}}^{2}}}{I_{L n i}} \times 100 \%=\frac{\sqrt{\sum_{k=2}^{50} I_{k_{n i}}^{2}}}{I_{L_{n i}}} \times 100 \%
\end{gathered}
$$

where $T H D_{V n i}$ is the total harmonic distortion of the voltage in phase $n$ at time $i, T D D_{I n i}$ is the total demand distortion of the current in phase $n$ at time $i, V_{m_{n i}}$ is the root mean square of the voltage for harmonic order $m$ in phase $n$ at time $i$ and $I_{m_{n i}}$ is the root mean square of the current for harmonic order $m$ in phase $n$ at time $i$.

$$
E_{96}=E_{d a y}=\sum_{t=1}^{T=96}\left(P_{L 1 t}+P_{L 2_{t}}+P_{L 3_{t}}\right)
$$

where $E_{k}$ is the energy consumption for $k$ time units during one day and $P_{n i}$ is the maximum power consumption in phase $n$ at time $i$.

The GA evaluates all fitness functions with respect to the objective function to find the Pareto Optimal solutions for this multi-objective problem. Via weighting factors for the fitness functions, the solutions can be shifted in the best case on the Pareto Frontier or at any rate in the solution space to emphasize specific optimization objectives.

According to (4), the objective function $\sigma$ translates the fitness functions into decision variables for a minimization $\left(T H D_{V}, T D D_{i}\right)$, respectively, and maximization $\left(E_{n}\right)$ problem.

$$
\begin{gathered}
\sigma_{i}(\Gamma)=\min . / \max .\left(\omega_{i} \Gamma_{i}\right) \\
\text { s.t. } \Gamma \epsilon\left(T H D_{V}, T D D_{I}, E_{n}\right)
\end{gathered}
$$

where $\Gamma_{i}$ represents the fitness/score values and $\omega_{i}$ the weights to emphasize the objectives.

\subsubsection{Selection Scheme}

The NSGA-II-non-dominated sorting genetic algorithm II is used in this approach to sort the solution space and determine the Pareto Frontier of the Pareto optimal solutions [58]. The selection operator used in NSGA-II is a binary tournament selector with the selection criterion based on the crowded-comparison operator $\prec_{n}$.

The crowded-comparison operator $\prec_{n}$ is defined as follows: 
Assume that every individual in the population has two attributes:

(1) Non-Dominated Rank,

(2) Crowding Distance.

Then $a \prec_{n} b$ is true, if $\left(a_{\text {rank }}<b_{\text {rank }}\right)$ or $\left(a_{\text {rank }}=b_{\text {rank }}\right.$ and $\left.a_{\text {distance }}>b_{\text {distance }}\right)$.

This means, in this case, the individual with the lower/better rank, which resembles lower THD and higher power values, is preferred in the evolutionary process. The next population will be selected following this principle. Therefore, the individual with the better non-dominated rank (which corresponds to a smaller rank value with 0 as the fittest) for $T H D_{V} / T D D_{i}$ and drawn energy $E_{n}$ is decorated with a higher priority. If two individuals share an identical non-dominated rank, those with a larger Crowding Distance are chosen to preserve diversity of the solutions and a uniformly spread out Pareto Frontier. To calculate the Crowding Distance, the distance between the fitness values in the solution space is evaluated.

\subsubsection{Mutation, Crossover and Validity Filter}

Unique to the mutation and the crossover scheme used in this investigation is the implementation of an additional validity filter. This patch function ensures computational efficiency because it prevents most invalid individuals from taking part in the evolutionary process.

The mutation scheme is based on the uniform mutation. Individuals are mutated by replacing random genes with uniformly distributed integer values within the predefined value range. In order to cover all the operation modes of each machine, the value range is adapted according to the state transition diagram. The flow chart of the modified mutation scheme including the patch function for validity check is represented in Figure 11a.

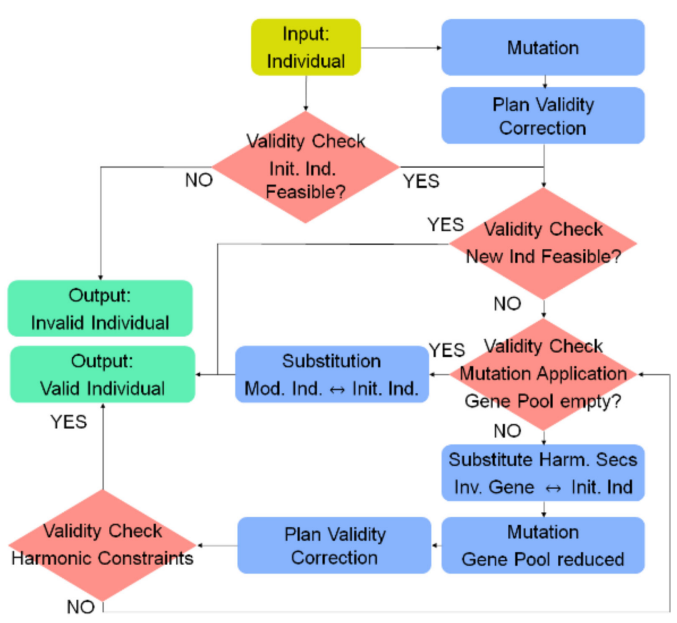

(a)

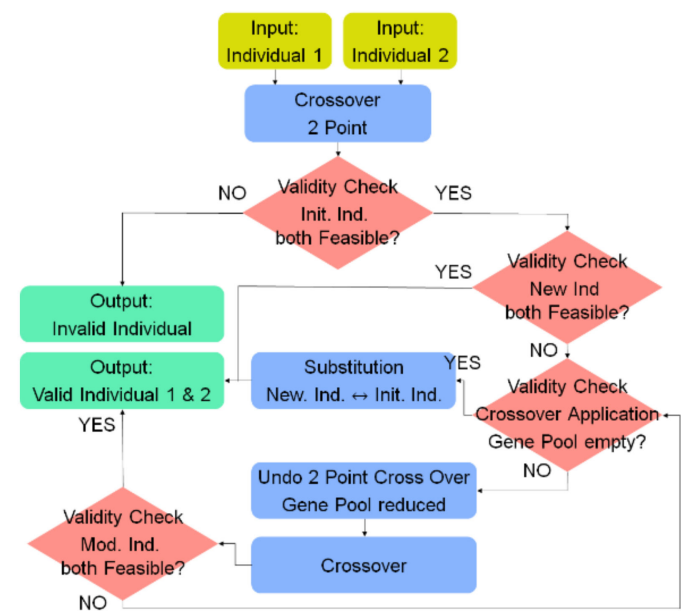

(b)

Figure 11. (a) Flow chart of the mutation scheme including the validity check and the patch function for plan validity correction. (b) Flow chart of the crossover scheme including the validity filter for the two initial and the two modified individuals.

The crossover scheme is based on the two-point crossover, which is applied by randomly selecting two points in the gene sequence of two individuals, indicating start and end point and exchanging these segments. The flow chart of the crossover scheme is illustrated in Figure 11b.

A third validity filter is applied to the initialization of the individuals to sort out invalids right at the beginning of the evolutionary process. This patch function follows a two-step scheme, beginning with the plan validity correction, as shown in Figure 12a, followed by a machine specific harmonic tuning function to further correct invalid individuals, as outlined in Figure 12b. 


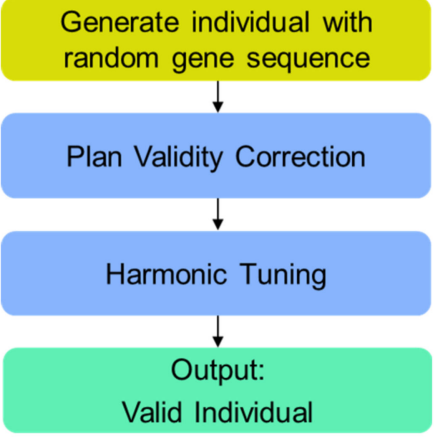

(a)

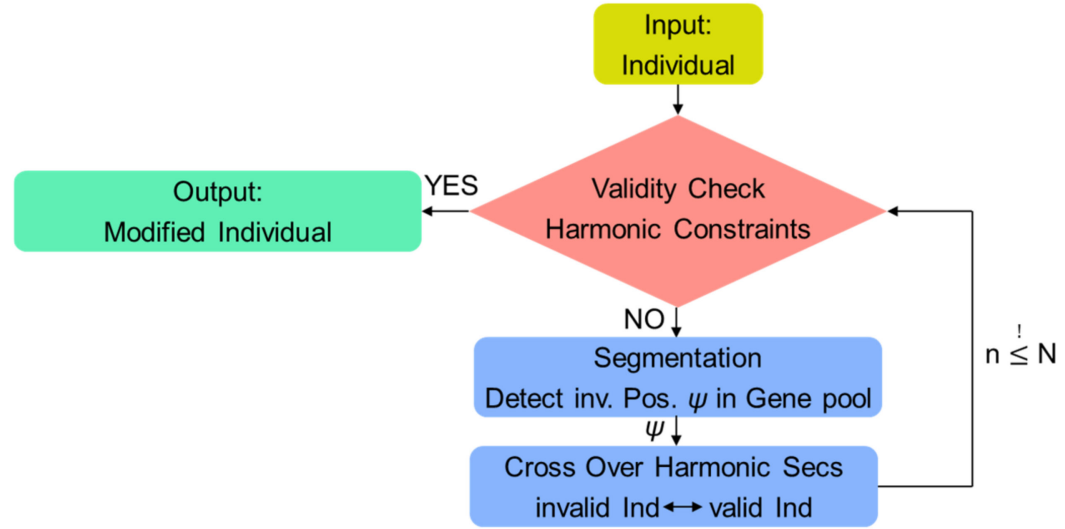

(b)

Figure 12. (a) Flow chart of the initialization operator for the first generation of individuals including the patch functions for plan validity correction and harmonic tuning. (b) Flow chart of the patch function for harmonic tuning.

\subsubsection{Penalty Function}

Due to the high cardinality of the individuals and the complexity of the boundary constraints $\Omega_{k}$, there is a high probability for invalid generations of the individuals and the options for invalidity filtering via the penalty function are limited. By adding a patch function into the initial generation unit, as well as the mutation unit and the crossover unit, it is ensured that most of the individuals taking part at the evolution process are feasible. However, there are still some possibilities that some of the generated individuals cannot be corrected. Therefore, the penalty function in this approach is mainly used as a secondary detection of invalid individuals. The proposed penalty function $\rho$ consists of two parts, the validity detection and the harmonic violation detection.

$$
\rho(\Gamma) \begin{cases}0 \text { for } M= & \Gamma \mid \Omega_{k} \\ \infty \text { for } N= & \Gamma \notin M\end{cases}
$$

where $\Omega_{k}$ represents the boundary conditions or physical constraints,

According to Equation (5), the invalid individuals will be penalized with a bad fitness value and, in this way, be sorted out in the next generations of the evolutionary process.

This further enhances the efficiency of the algorithm by filtering infeasible solutions, which could not be corrected by the patch functions applied to the initialization and mutation operator.

\subsection{Human Machine Interface}

A human machine interface (HMI) was implemented with PyQT to combine the scheduling of the machines/designation of flexibilities by the plant operator with the optimization algorithm of the AI in the backend. The goal of the development of the HMI was therefore to integrate the interaction of the administrative plant operator and the optimization algorithm into a graphical user interface. The following core elements were considered in the development:

- Creation and adjustment of the operating plans,

- Configuration of the optimization,

- Visualization of the optimization result.

Schedules are created for each facility per day, with 96 (15 min) blocks per load. This time segmentation is based on reasonable resource planning for operators as well as providing an interface for the time schedule of the power exchange, at which the intra-day market products are considered prospectively. The operator has access to the three options 'Off', 'On' and 'Flexible'. The operating plans can be created arbitrarily far into the future 
and can be modified at any time until the optimization is started. Once the optimization has been completed, the created schedules are displayed to the operator for final operational adjustments and stored in the database. Basically, a specific system model is used for the optimization phase, adaptable to any industrial process chain. It has to be stated that adjustments to the digital twin of the physical system have to be made in this step for every change in the system, which could, for instance, mean a change in the infrastructure of the electrical grid.

Figure 13 shows two main windows of the HMI. The machine pool and the predefined schedules can be overseen here. Figure 13a shows the planning scheme for the available industrial loads, Figure $13 \mathrm{~b}$ the evaluation mask for the calculated grid state. Each load can be included into the scheduling after an electrical footprint has been made by measurements.

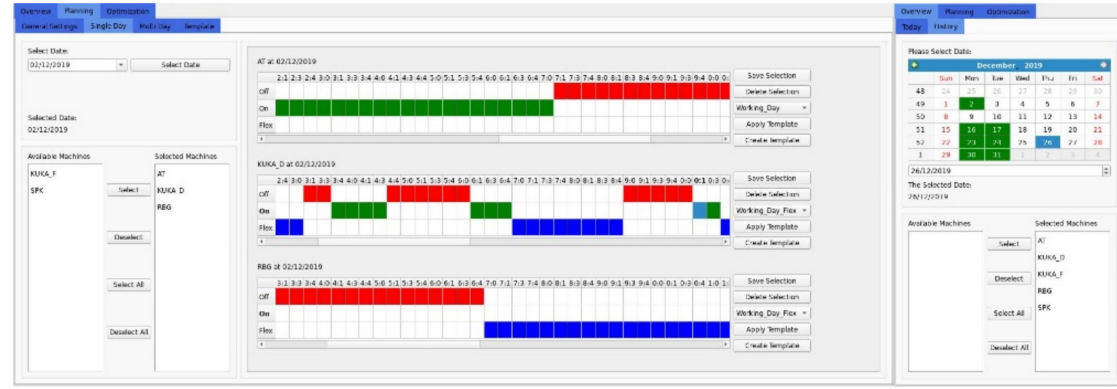

(a)

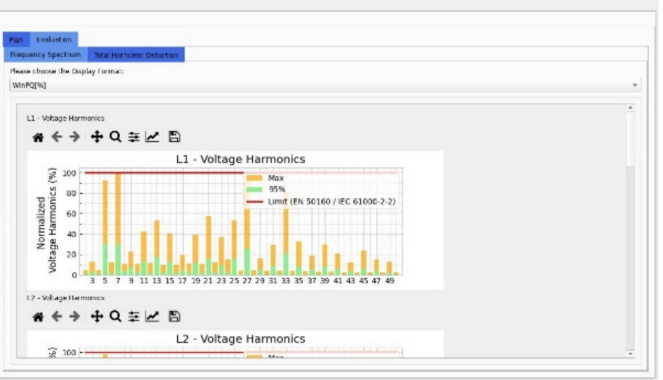

(b)

Figure 13. (a) Human Machine Interface for the intelligent process planning scheme with flexibility declaration. (b) Interface of the results for the simulated grid state including the designated, controlled machine pool.

A main reason for implementing the HMI was the fact that, in real-life scenarios, not all machines may be scheduled at all time steps. Practicability must be accounted for while still allowing the $\left(T H D_{V} / T D D_{i}\right)$ to be lowered to allow the (human) plant operator to set the flexibility options for each machine. If a machine cannot be scheduled, it may be excluded from the optimization process by simply not declaring it as a flexibility. It still can be considered as a harmonic source by setting it as fixed in operation at a certain operating point for each time step.

\section{Results}

\subsection{Benchmark of Data Stores}

For a performance comparison of the DBs which hold the necessary data for the algorithm, a container is created and filled with a total of 100,000,000 values, which corresponds to a total of 1000 signals at a sampling rate of 500,000 samples per second and 10 periods per signal. The benchmarked DBs were used in the following versions:

- $\quad$ MongoDB: Ver. 3.6

- InfluxDB: Ver. 1.5.2

- $\quad$ PostgreSQL: Ver. 10.3

The data were first generated with a Python script and stored in various file formats for testing. The Python version in use was 3.4 with pickle protocol 0.

The pickle module saves the data compressed in files, depending in detail on the protocol in use. The memory space of the file without time information and without further tags is $78.2 \mathrm{MB}$. By choosing protocol 0 , the data are saved in a readable format but need about $62 \%$ more memory, thus occupying $127 \mathrm{MB}$. This also corresponds approximately to the file size of a CSV file, which is also directly accessible by the user. However, all of these files lack the time information which is later concatenated in a helper function, which derives the time stamp from the information of the sample rate. If the data are saved with a 
time stamp and in a human readable format, this results in a file size of $200 \mathrm{MB}$, compared to the pickle file, with a size of $123 \mathrm{MB}$.

After importing the data in MongoDB, the total size of the collection with the signals is $353.6 \mathrm{MB}$ without further information being stored besides the time stamp and the signal values.

InfluxDB has a much higher compression and needs a total of $68.7 \mathrm{MB}$ for the data. Additional storage capacity is required for the metadata and the respective time stamp. Thus, InfluxDB has a memory requirement of approximately $102 \mathrm{MB}$.

In contrast to the other two databases, PostgreSQL first has to create a new table with a predefined structure. After storing the signals, the table occupies a disk space of $806 \mathrm{MB}$; the total database with this single table $1027 \mathrm{MB}$. PostgreSQL intrinsically causes some overhead with varying table structures because all fields have to be filled with a value, which occupies space without bearing additional information because of the predefined structure of the tables.

Influx $D B$ thus requires the least storage capacity, as shown in Figure 14, and is best suited for a very high volume of data.

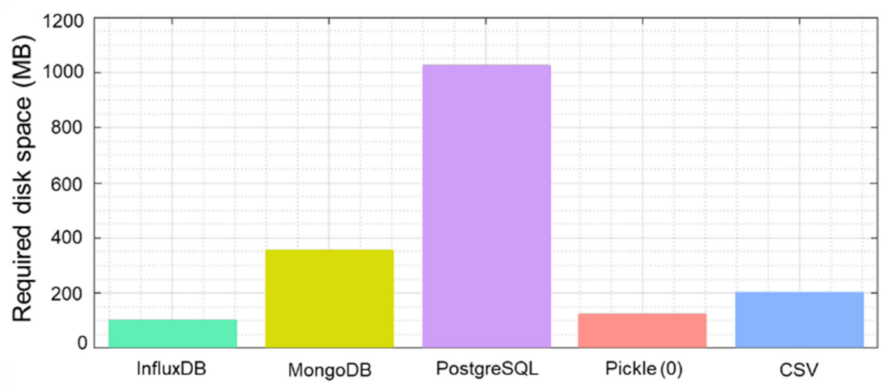

Figure 14. Required disk space for the different storage types for a dataset with $10^{6}$ data points plus time stamp. InfluxDB shows the highest compression rate for those kinds of data.

For the test of maximum write operations, the data series is comparatively written into the databases one by one and in batch packages. It can be observed that the batch processing performs much faster. Yet, this option is not available for all databases. $\operatorname{InfluxDB}$ offers a multi-point writing feature in the Python module, being up to 500 times faster than writing each point individually. For individual processing, 1000 fields per second could be written, or in case of batch processing, up to 503,000 fields per second.

PostgreSQL also offers a much faster way of storing data from files in the database using the COPY method, which is suitable for multiple datasets. However, this method has the disadvantage that the order of the files, e.g., for comma-separated values (CSV) files, must be exactly the same as the table structure. Otherwise, the database stores the values in a wrong topology or aborts the process with an error code. For this reason, the COPY method is not suitable for the proposed use case. An example would be the displacement of all data by a certain number of columns or rows, due to different lengths of the arrays of the measurement data. Thus, each row of a data source has to be read out individually and stored separately with a SQL command in the PostgreSQL database. This method ensures that all data exist in the correct format and table size. In this test, MongoDB reaches the highest score with up to 531,000 fields per second. When writing single points, MongoDB was slightly slower than InfluxDB with 890 fields per second. Figure 15a,b show that several measurement series should always be stored together and, therefore, be processed as a batch. 


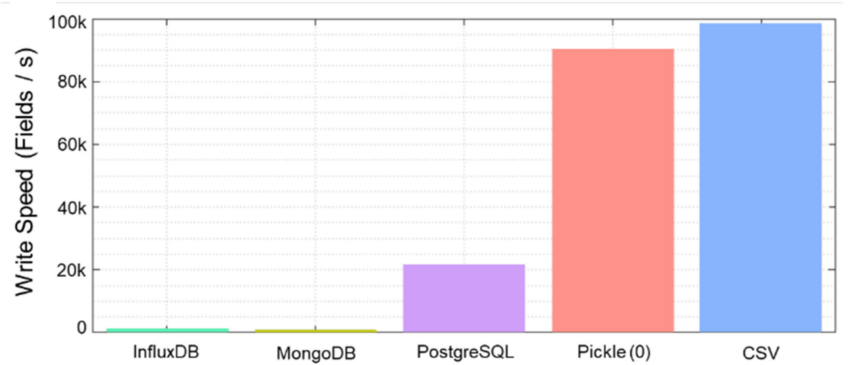

(a)

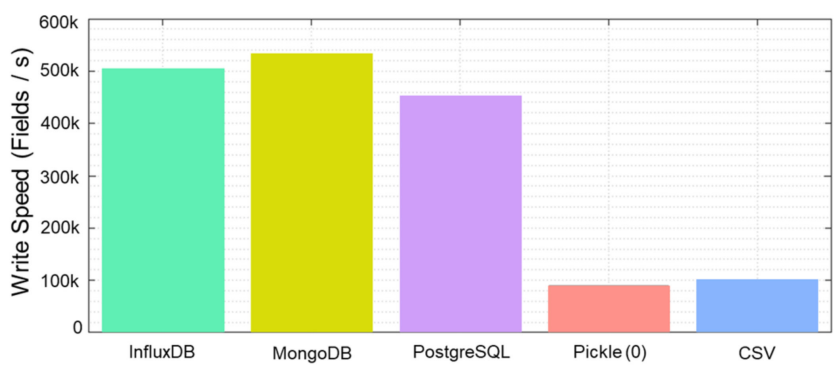

(b)

Figure 15. (a) Written fields per second for single field processing. (b) Written fields per second for accumulated batch processing.

In conclusion, InfluxDB and MongoDB outperform PostgreSQL in the proposed use case because of the intrinsic constraints, accompanying the concept of a relational DB. InfluxDB shows advantages in compression rate and query speed and comes just a little short in terms of writing speed.

\subsection{Smart Demand-Side-Management}

Two parameters- $T H D_{V}$ and $T D D_{i}$-are targeted as the objective minimization function for the GA to optimize the operational schedules for a lower harmonic distortion in the industrial grid. The second optimization objective aims for a maximization of the utilization in all 96 time units during the day, if no restricting boundary conditions are set.

Two scenarios were investigated to, first, provide a proof of concept for the proposed methodology and, second, to demonstrate the applicability to a modern industrial environment. Therefore, a simplified application for a synthesized test case with a predefined optimal solution was designed. Following this, the methodology was extended to a small industrial grid with five different machines.

\subsubsection{Proof of Concept}

A test case was synthesized to prove the validity of the developed algorithmic. Therefore, the optimal scheduling of a virtual machine with a same state machine such as the AGV (see Figure 1b) is investigated.

The mean voltage harmonics from 2 nd to 50th degree, resulting from the virtual machine in operation mode 3, are set to $1 / 5$ of the limits, according to EN 50160-2015 and IEC 61000-2-2. The permissible limits for this test case were set to $1 / 6$ of the normative limits, to be below the harmonic emission level of the machine at its highest operation mode. This results in a permissible $T H D_{V}$ value (2nd-50th harmonic) of $8.121 \% \times 1 / 6=$ $1.354 \%$ (combining the limits for individual harmonics of EN 50160 + IEC 61000-2-2 leads to a $T H D_{V}$ limit of $8.121 \%$ ).

Virtual start-up harmonics that arise when switching from state 'off' to 'on' are assigned to double the limits and are superimposed to the frequency spectrum of normal operation. Consequently, if working correctly, the algorithm tries to avoid such switching operations. The power of each state is varying and set to ten times the state number, i.e., $0 \mathrm{~W}$ for state $0,10 \mathrm{~W}$ for state $1,20 \mathrm{~W}$ for state 2 and $30 \mathrm{~W}$ for state 3 .

The boundary constraints are composed of two parts, the voltage harmonic limitations and the physical constraints, according to the state machine.

The objectives for the optimization are maximization of power and minimization of $T H D_{V}$ for voltage in each phase. This definitions lead to a global optimum with a drawn energy of $2.160 \mathrm{kWh}$ and a maximal $T H D_{V}$ of $1.354 \%$. The results for a varied number of generations and population sizes are shown in Table 3. 
Table 3. Results of the proof of concept for a virtual machine in a predefined scenario. Marked in light-/green are the local respective global optima found by the algorithm.

\begin{tabular}{ccccc}
\hline Generations & Pop. Size & $\boldsymbol{E}_{\text {day }}$ & THDV & Runtime \\
\hline 100 & 1000 & $1.65 \mathrm{kWh}$ & $0.9329 \%$ & $1469.50 \mathrm{~s}$ \\
100 & 5000 & $1.76 \mathrm{kWh}$ & $1.3254 \%$ & $8279.53 \mathrm{~s}$ \\
100 & 10,000 & $1.82 \mathrm{kWh}$ & $1.3536 \%$ & $13,172.46 \mathrm{~s}$ \\
100 & 20,000 & $1.93 \mathrm{kWh}$ & $1.3536 \%$ & $26,431.36 \mathrm{~s}$ \\
500 & 1000 & $2.07 \mathrm{kWh}$ & $1.3536 \%$ & $8100.78 \mathrm{~s}$ \\
800 & 1000 & $2.16 \mathrm{kWh}$ & $1.3536 \%$ & $13,555.90 \mathrm{~s}$ \\
1000 & 1000 & $2.16 \mathrm{kWh}$ & $1.3536 \%$ & $15,393.44 \mathrm{~s}$ \\
2000 & 1000 & $2.16 \mathrm{kWh}$ & $1.3536 \%$ & $32,157.59 \mathrm{~s}$ \\
\hline
\end{tabular}

As can be derived from Table 3, with an increasing number of generations and population size, the exploration range has been expanded, which results in better fitness values closer to the global optimum. Having reached the vicinity of the global optimum, the solutions do not diverge or deteriorate, due to the elitist selection strategy of the NSGA II.

Therefore, it can be assumed the proposed approach converges to the global optimum if the exploration range is large enough. This gave confidence to apply the approach to a more complex grid with different types of machines.

\subsubsection{Case Study-Industry 4.0 Application}

For the case study of a realistic industrial application, the five machines introduced in Section 2.2 were all designated as flexibilities during a usual working day. This means that the optimization algorithm may set the operating point for each machine, respecting the implemented physical boundary conditions, defined by the respective state machine (Section 2.1). For reference, the same setup was simulated with all machines in a fully utilized mode over the same period. The schedule for the reference and the Smart DSM showcase is shown in Table 4. The applied objective function aims for a minimization of the $T H D_{V}$ in all three phases whilst maximizing the utilization of the plant by increasing the amount of drawn energy by the system. Figure 16 shows the results for phase one of the industrial grid, measured at the point of common coupling of the industrial loads. The results illustrate the improvement for the harmonic levels, superimposed on a virtual point of common coupling (PCC), where all the machines are connected to the grid. Figure 16a gives the results for the reference system, with all machines on full operation for the whole working day. The working day starts at 07:00 a.m. and ends at 05:00 p.m. The power trend shows a maximum power demand of about $30 \mathrm{~kW}$ with a small excitation at 07:00 a.m., resulting from inrush currents of the machines. The associated high frequency components are not reflected in the $T H D_{V}$ and the $T D D_{I}$ due to the averaging interval of $15 \mathrm{~min}$ in this representation, resulting from the scheduling interval. Excitations of the normative limits could be stated for several key factors, as listed in Table 5, especially the $T H D_{V}$, the $T D D_{I}$, the 5 th, 7 th, 27 th and 33th harmonic. Figure $16 \mathrm{~b}$ shows the results for the optimized grid. The frequency spectrum for both cases points to the characteristic harmonic weighting accompanying B6 bridge circuits. As can be concluded from Figure 16 and Table 5, the Smart DSM is capable of optimizing even complex structures with multiple (physical) boundary constraints and conflicting objectives. Table 5 further illustrates the improvement, particularly for $T H D_{V}$, which was designated as the primary optimization objective. The timeline of the $T H D_{V}$ as well as the maximum/95th percentile values found good agreement with the configuration, allowing short termed excitation of the limits (one time step) to further enhance the utilization factor. Despite this additional degree of freedom, the algorithm found good solutions for the designated schedule, as all the single harmonics met their normative limits for the whole period. The main objective of lowering the $T H D_{V}$ to normative limits was met in accordance with expectations, with the convergence speed and degrees of freedom adjustable with the configuration of the optimization. 
Table 4. Schedule for the reference case of the industrial grid with all loads in full operation mode and the optimized case via the Smart DSM approach with all machines marked as flexibilities during the working day. In 'off' state all machines are switched off, in 'on' state all machines are turned on and in 'flex' state the optimization algorithm decides, which operation state is to be chosen.

\begin{tabular}{cccc}
\hline & \multicolumn{2}{c}{ Reference } & \multicolumn{2}{c}{ Smart DSM } \\
\hline 'off' state & from 00:00-07:00 & 'off' state & from 00:00-07:00 \\
'on' state & from 07:00-17:00 & 'flex' state & from 07:00-17:00 \\
'off' state & from 17:00-24:00 & 'off' state & from 17:00-24:00 \\
\hline
\end{tabular}
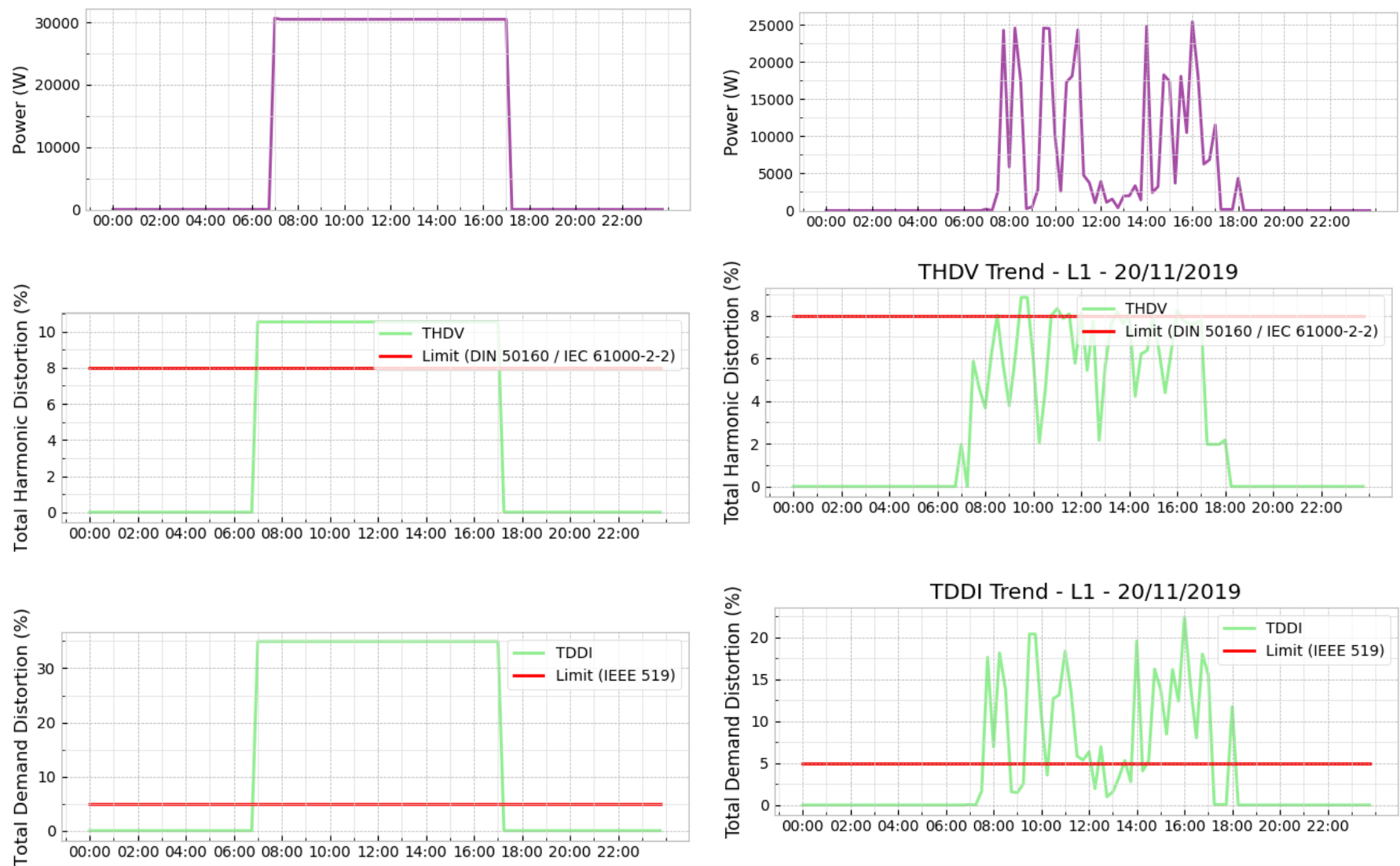

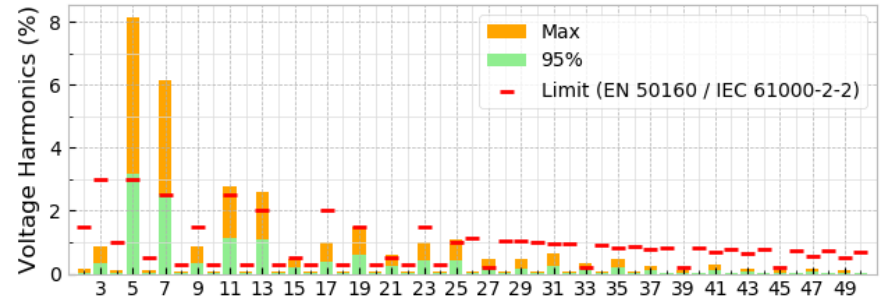

(a)

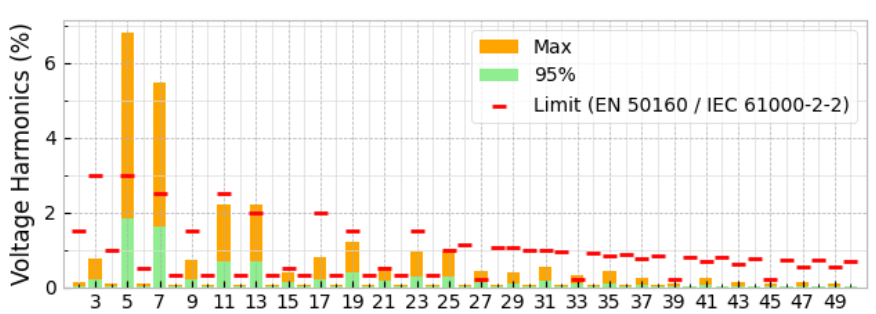

(b)

Figure 16. (a) Results for the reference case for the industrial machine pool on full utilization during the whole working day. (b) Results for the optimized utilization of the machine pool via the Smart DSM algorithm. All machines are connected at a virtual point of common coupling to the grid. The Smart DSM algorithm was allowed short excitations of the normative limits to further enhance the convergence speed. All key indexes showed improvements, pointing towards the Pareto optimal Frontier to maintain a safe grid state in accordance with the normative limits while still ensuring a high utilization. 
Table 5. Excitations of the normative limits for the reference case and the optimized grid for the industrial application. Marked in red are the normative limit violations (for harmonics and $T H D_{V}$ from EN 50160/61000-2-2, for $T D D_{I}$ from IEEE 519) for the 95th percentile of the values during the time interval of one day, in yellow the limit violations only for the maximum values and in green the values in accordance to their respective limits. The $T H D_{V}$ was the main objective of the optimization.

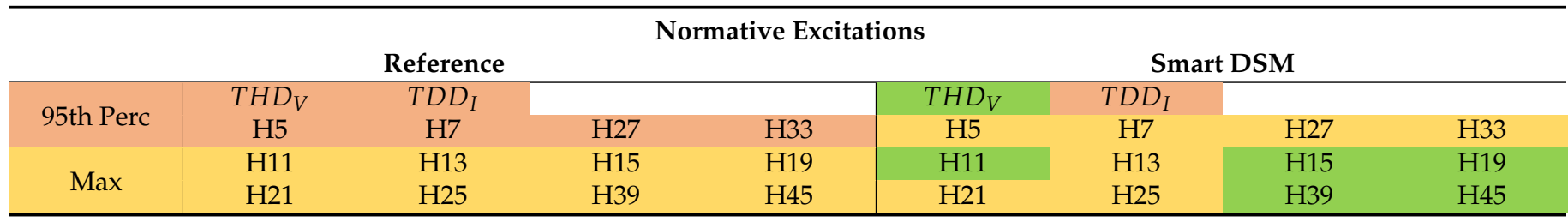

\section{Conclusions}

In this paper, a PQ mitigation method based on DSM and a GA is proposed. In a proof of concept, it was shown that the NSGA-II algorithm is applicable for an optimal scheduling of industrial processes. With the proposed optimization method, machines can operate on a low harmonic distortion level, reducing extra losses while ensuring a high productivity.

The proposed approach is implemented as a load management application with complex boundary conditions to avoid critical grid states. Moreover, due to the possibility of producing unqualified solutions in the evolutionary process restricted by the physical boundary conditions, patch functions are added to each operation module of the algorithm. This way, only feasible individuals are allowed to participate in the evolution process, thereby improving the overall optimization efficiency and the grid state. Due to the straightforward formulation of the fitness functions, the optimization is well designed for extensibility for multiple objectives.

This optimization algorithm is specifically suitable for industrial grids as a tool for high complexity problems such as intelligent determination of operational schedules for a machine pool. An HMI links the site operator and the scheduling/optimization algorithm for the demand side. It enables the operator to define the flexibility of different machines according to their requirements. The plant operator therefore retains decision-making sovereignty by defining the degrees of freedom of optimization. Based on the definition of the flexibilities, the optimization of the state space of the machines can be performed by the algorithm, thus mitigating the harmonic distortion and therefore improving the grid state. Parameters evaluating the grid state such as active/reactive/apparent power, $T H D_{V}$ and $T D D_{I}$ may be used as fitness parameters to participate in the survival of the fittest during the evolution process as selectable objective functions.

Further potential investigations resulting from this paper are the analysis of the transferability of the approach to different grid structures, especially for the scaling effects of the computational needs and the comparison with alternative approaches such as sophisticated deep learning methods, handling the same environment.

The scaling potential of the algorithm is especially of interest for the optimization of extended grid structures, such as large industrial sites with multiple machines of different types and applications. Since the GA algorithm is known for a high computational cost, these scaling effects have to be considered when projecting the methodology to large real-life applications. Measures for software- and hardware-wise optimization such as parallelization and an extended cluster structure bear huge potential for further design optimizations.

Methodology-wise, the information and communication infrastructure may be additionally considered to map the influence of the signal runtime of sensors and actuators on the mitigation of harmonics. 


\begin{abstract}
Author Contributions: Conceptualization, A.E.; methodology, A.E.; software, A.E.; validation, A.E., T.S. and K.R.; formal analysis, A.E.; investigation, A.E.; resources, A.E.; data curation, A.E.; writingoriginal draft preparation, A.E.; writing—review and editing, A.E., T.S. and K.R.; visualization, A.E.; supervision, K.R.; project administration, K.R.; funding acquisition, A.E. and T.S. All authors have read and agreed to the published version of the manuscript.
\end{abstract}

Funding: This publication was supported by the German Research Foundation (DFG) within the funding programme Open Access Publishing.

Institutional Review Board Statement: Not applicable

Informed Consent Statement: Not applicable.

Data Availability Statement: No publicly archived data sets were analyzed.

Conflicts of Interest: The authors declare no conflict of interest.

\title{
References
}

1. Bollen, M.H.; Gu, I. Signal Processing of Power Quality Disturbances; John Wiley \& Sons: Hoboken, NJ, USA, 2006; p. 30. ISBN 0471931306.

2. Currence, E.J.; Plizga, J.E.; Nelson, H.N. Harmonic resonance at a medium-sized industrial plant. IEEE Trans. Ind. Appl. 1995, 31, 682-690. [CrossRef]

3. Dugan, R.C.; McGranaghan, M.F.; Beaty, H.W. Electrical Power Systems Quality; McGraw-Hill: New York, NY, USA, 1996.

4. IEEE Working Group on Power System Harmonics. Power system harmonics: An overview. IEEE Trans. Power Appar. Syst. 1983, $8,2455-2460$.

5. Wang, X.; Blaabjerg, F. Harmonic stability in power electronic-based power systems: Concept, modeling, and analysis. IEEE Trans. Smart Grid 2018, 10, 2858-2870. [CrossRef]

6. Messo, T.; Luhtala, R.; Aapro, A.; Roinila, T. Accurate impedance model of a grid-connected inverter for small-signal stability assessment in high-impedance grids. IEEJ J. Ind. Appl. 2019, 8, 488-496. [CrossRef]

7. Shah, S.; Koralewicz, P.; Gevorgian, V.; Wallen, R.; Jha, K.; Mashtare, D.; Burra, R.; Parsa, L. Large-signal impedance-based modeling and mitigation of resonance of converter-grid systems. IEEE Trans. Sustain. Energy 2019, 10, 1439-1449. [CrossRef]

8. Chakravorty, D.; Meyer, J.; Schegner, P.; Yanchenko, S.; Schocke, M. Impact of modern electronic equipment on the assessment of network harmonic impedance. IEEE Trans. Smart Grid 2016, 8, 382-390. [CrossRef]

9. Hanzelka, Z.; Bien, A. Power Quality Application Guide: Harmonics E Interharmonics. A Guide Material by Leonardo Power Quality Initiative; Copper Development Association: New York, NY, USA, 2004.

10. Das, J.C. Power System Harmonics and Passive Filter Designs; John Wiley \& Sons: Hoboken, NJ, USA, 2015.

11. Riccobono, A.; Liegmann, E.; Monti, A.; Dezza, F.C.; Siegers, J.; Santi, E. Online wideband identification of three-phase AC power grid impedances using an existing grid-tied power electronic inverter. In Proceedings of the 2016 IEEE 17th Workshop on Control and Modeling for Power Electronics (COMPEL), Trondheim, Norway, 27-30 June 2016.

12. Bollen, M.H.; Zhong, J.; Zavoda, F.; Meyer, J.; McEachern, A.; Córcoles López, F. Power quality aspects of smart grids. In Proceedings of the International Conference on Renewable Energies and Power Quality (ICREPQ’10), Granada, Spain, 23-25 March 2010.

13. Meyer, J.; Klatt, M.; Schegner, P. Power quality challenges in future distribution networks. In Proceedings of the 20112 nd IEEE PES International Conference and Exhibition on Innovative Smart Grid Technologies, Manchester, UK, 5-7 December 2011.

14. Bollen, M.H. Understanding Power Quality Problems; IEEE Press: Piscataway, NJ, USA, 2000.

15. Arrilaga, J.; Wattson, N.R.; Chen, S. Power System Quality Assessment; Wiley: Hoboken, NJ, USA, 2000.

16. Singh, B.; Chandra, A.; Al-Haddad, K. Power Quality: Problems and Mitigation Techniques; Wiley: Hoboken, NJ, USA, 2014.

17. Porter, G.J.; Sciver, J.A.E. Power Quality Solutions: Case Studies for Trouble Shooters; The Fairmount: Lilburn, GA, USA, 1999.

18. Heydt, G.T. Electric Power Quality; Stars in a Circle: West Lafayette, IN, USA, 1994.

19. IEEE. IEEE Guide for Application and Specification for Harmonic Filters. IEEE Stand. 2003, 1531, 1-60.

20. Das, J.C. Passive filter-potentialities and limitations. IEEE Trans. Ind. Appl. 2004, 40, 232-241. [CrossRef]

21. Ghosh, A.; Ledwich, G. Power Quality Enhancement Using Custom Power Devices; Springer Science \& Business Media: Norwell, MA, USA, 2002.

22. Nastran, J.; Cajhen, R.; Seliger, M.; Jereb, P. Active power filters for Nonlinear AC loads. IEEE Trans. Power Electron. 1994, 9, 92-96. [CrossRef]

23. Ritwik, M. Reactive Power Compensation in Single-Phase Operation of Microgrid. IEEE Trans. Ind. Electron. 2013, 60, 1403-1416.

24. Dou, J.; Ma, H.; Yang, J.; Zhang, Y.; Guo, R. An Improved Power Quality Evaluation for LED Lamp Based on G1-Entropy Method. IEEE Access 2021, 9, 111171-111180. [CrossRef]

25. Iqbal, M.N.; Kütt, L.; Daniel, K.; Jarkovoi, M.; Asad, B.; Shabbir, N. Bivariate stochastic model of current harmonic analysis in the low voltage distribution grid. Proc. Est. Acad. Sci. 2021, 70, 190. 
26. Daniel, K.; Kütt, L.; Iqbal, M.N.; Shabbir, N.; Rehman, A.U.; Shafiq, M.; Hamam, H. Current Harmonic Aggregation Cases for Contemporary Loads. Energies 2022, 15, 437. [CrossRef]

27. Eisenmann, A.; Streubel, T.; Rudion, K. General Framework for Simulating Power Quality Data Processing. In Proceedings of the 26th International Conference \& Exhibition on Electricity Distribution-CIRED, Online, 20-23 September 2021.

28. Saxena, D.; Verma, K.; Singh, S. Power quality event classification: An overview and key issues. MultiCraft Int. J. Eng. Sci. Technol. 2010, 2, 186-199. [CrossRef]

29. Mohan, N.; Soman, K.P.; Vinayakumar, R. Deep power: Deep learning architectures for power quality disturbances classification In Proceedings of the 2017 International Conference on Technological Advancements in Power and Energy (TAP Energy), Kollam, India, 21-23 December 2017.

30. Stuchly, J.; Misak, S.; Vantuch, T.; Burianek, T. A power quality forecasting model as an integrate part of active demand side management using artificial intelligence technique-Multilayer neural network with backpropagation learning algorithm. In Proceedings of the 2015 IEEE 15th International Conference on Environment and Electrical Engineering (EEEIC), Rome, Italy, 10-13 June 2015; pp. 611-616.

31. Eisenmann, A.; Streubel, T.; Rudion, K. PQ classification by way of parallel computing-A sensitivity analysis for a real-time LSTM approach using waveform and RMS data. In Proceedings of the 13th IEEE PES PowerTech Conference, Milano, Italy, 23-27 June 2019.

32. Eisenmann, A.; Streubel, T.; Rudion, K. PQ prediction by way of parallel computing - benchmark and sensitivity analysis for classical ML approaches. In Proceedings of the 25th International Conference \& Exhibition on Electricity Distribution-CIRED, Madrid, Spain, 3-6 June 2019.

33. Eisenmann, A.; Streubel, T.; Rudion, K. Power Quality Prediction by way of Parallel Computing-A New Approach Based on a Long Short-Term Memory Network. In Proceedings of the 2019 IEEE PES Innovative Smart Grid Technologies Europe (ISGT Europe 2019), Bucharest, Romania, 29 September-2 October 2019.

34. Eisenmann, A.; Streubel, T.; Rudion, K. An Investigation on feature extraction and feature selection for power quality classification with high resolution and RMS data. In Proceedings of the 9th International Conference on Renewable Power Generation-IET RPG, Dublin, Ireland, 1-2 March 2021.

35. Eisenmann, A.; Streubel, T.; Rudion, K. Manual Configuration and Best Setup of Support Vector Machines for Power Quality Classification. In Proceedings of the 14th IEEE PES PowerTech Conference, Madrid, Spain, 27 June-2 July 2021.

36. Qaisar, S.M.; Aljefri, R. Effective Power Quality Disturbances Identification Based on Event-Driven Processing and Machine Learning. Green Energy Sol. Energy Photovolt. Smart Cities 2020, 191-219. [CrossRef]

37. Saucedo-Dorantes, J.J.; Elvira-Ortiz, D.A.; Jaen-Cuéllar, A.Y.; Toledano-Ayala, M. Novelty Detection Methodology Based on SelfOrganizing Maps for Power Quality Monitoring. In Artificial Intelligence-Latest Advances, New Paradigms and Novel Applications; IntechOpen: London, UK, 2021. [CrossRef]

38. Ernst, D.; Glavic, M.; Wehenkel, L. Power systems stability control: Reinforcement learning framework. IEEE Trans. Power Syst. 2004, 19, 427-435. [CrossRef]

39. Kara, E.C.; Berges, M.; Krogh, B.; Kar, S. Using smart devices for system-level management and control in the smart grid: A reinforcement learning framework. In Proceedings of the 2012 IEEE Third International Conference on Smart Grid Communications (SmartGridComm), Tainan, Taiwan, 5-8 November 2012; pp. 85-90.

40. Bagheri, M.; Nurmanova, V.; Abedinia, O.; Salay Naderi, M. Enhancing power quality in microgrids with a new online control strategy for dstatcom using reinforcement learning algorithm. IEEE Access 2018, 6, 38986-38996. [CrossRef]

41. Ruelens, F.; Claessens, B.J.; Vandael, S.; Iacovella, S.; Vingerhoets, P.; Belmans, R. Demand response of a heterogeneous cluster of electric water heaters using batch reinforcement learning. In Proceedings of the 2014 Power Systems Computation Conference, Wroclaw, Poland, 1-7 August 2014.

42. Sheikhi, A.; Rayati, M.; Ranjbar, A.M. Demand side management for a residential customer in multi-energy systems. Sustain. Cities Soc. 2016, 22, 63-77. [CrossRef]

43. François-Lavet, V.; Taralla, D.; Ernst, D.; Fonteneau, R. Deep Reinforcement Learning Solutions for Energy Microgrids Management. In European Workshop on Reinforcement Learning (EWRL 2016). 2016. Available online: https://orbi.uliege.be/ bitstream/2268/203831/1/EWRL_Francois-Lavet_et_al.pdf (accessed on 2 February 2019).

44. Mocanu, E.; Mocanu, D.C.; Nguyen, P.H.; Liotta, A.; Webber, M.E.; Gibescu, M.; Slootweg, J.G. On-line building energy optimization using deep reinforcement learning. IEEE Trans. Smart Grid 2019, 10, 3698-3708. [CrossRef]

45. Vikhar, P.A. Evolutionary algorithms: A critical review and its future prospects. In Proceedings of the 2016 International Conference on Global Trends in Signal Processing, Information Computing and Communication (ICGTSPICC), Jalgaon, India, 22-24 December 2016.

46. Yao, X. Global Optimization by Evolutionary Algorithms. IEEE Trans. Evol. Comput. 1999, 3, 82-102.

47. Miranda, V.; Srinivasan, D.; Proença, L.M. Evolutionary computation in power systems. Electr. Power Energy Syst. 1998, $20,89-98$. [CrossRef]

48. Awais, M.; Javaid, N.; Shaheen, N.; Iqbal, Z.; Rehman, G.; Muhammad, K.; Ahmad, I. An Efficient Genetic Algorithm Based Demand Side Management Scheme for Smart Grid. In Proceedings of the 2015 18th International Conference on Network-Based Information Systems, Taipei, Taiwan, 2-4 September 2015.

49. IEC. Electromagnetic compatibility (EMC)—Part 4-30: Testing and measurement techniques-Power quality measurement methods. In IEC 61000-4-30:2015; IEC: Geneva, Switzerland, 2015. 
50. Task Force on Harmonics Modeling and Simulation. Modeling and Simulation of the Propagation of Harmonics in Electric Power Networks. IEEE Transact. Power Delivery 1996, 11, 466-474. [CrossRef]

51. Schicker, E. Datenbanken und SQL, 5th ed.; Springer Vieweg: Wiesbaden, Germany, 2017.

52. Heuer, A.; Sattler, K.-U.; Saake, G. Datenbanken-Konzepte und Sprachen, 6th ed.; MITP: Frechen, Germany, 2018.

53. Studer, T. Relationale Datenbanken, 1st ed.; Springer Vieweg: Wiesbaden, Germany, 2016.

54. Meier, A. Werkzeuge der Digitalen Wirtschaft: Big Data, NoSQL E Co; Springer Vieweg: Wiesbaden, Germany, 2018.

55. IEC. Voltage characteristics of electricity supplied by public electricity networks. In EN 50160:2015, German version EN 50160:2010 + Cor.:2010 + A1:2015; Beuth: Berlin, Germany, 2015.

56. IEC. Electromagnetic compatibility (EMC)-Part 2-2: Environment-Compatibility levels for low-frequency conducted disturbances and signalling in public low-voltage power supply systems. In IEC 61000-2-2:2002 + A1:2017 + A2:2018, German version EN 61000-2-2:2002 + A1:2017 + A2:2019; IEC: Geneva, Switzerland, 2019.

57. IEC. IEEE Recommended Practice and Requirements for Harmonic Control in Electric Power Systems. In IEEE 519-2014; IEEE SA: Piscataway Township, NJ, USA, 2014.

58. Deb, K.; Pratap, A.; Agarwal, S.; Meyarivan, T. A Fast and Elitist Multiobjective Genetic Algorithm: NSGA-II. IEEE Trans. Evol. Comput. 2002, 6, 182-197. [CrossRef] 